R. Zhang, I. Boxx, W. Meier, C.D. Slabaugh, Coupled Interactions of a Helical Precessing Vortex Core and the Central Recirculation Bubble in a Swirl Flame at Elevated Power Density, Combustion and Flame 202 (2019) 119-131.

The original publication is available at www.elsevier.com

https://doi.org/10.1016/j.combustflame.2018.12.035

(C) 2019. This manuscript version is made available under the CC-BY-NC-ND 4.0 license http://creativecommons.org/licenses/by-nc-nd/4.0/ 


\title{
Coupled Interactions of a Helical Precessing Vortex Core and the Central Recirculation Bubble in a Swirl Flame at Elevated Power Density
}

\author{
Robert Zhang $^{\mathrm{a}}$, Isaac Boxx ${ }^{\mathrm{b}}$, Wolfgang Meier ${ }^{\mathrm{b}}$, Carson D. Slabaugh ${ }^{\mathrm{a}, *}$ \\ ${ }^{a}$ School of Aeronautics and Astronautics, Purdue University, 701 W. Stadium Ave., West Lafayette, IN \\ $47907-2045$ \\ ${ }^{b}$ German Aerospace Center (DLR), Institute of Combustion Technology, Pfaffenwaldring 38-40, 70569 \\ Stuttgart, Germany
}

\begin{abstract}
The PRECCINSTA GTMC was studied at elevated pressure and power density with $6 \mathrm{kHz}$ stereoscopic particle image velocimetry (SPIV), $\mathrm{OH}^{*}$ chemiluminescence (CL), and $100 \mathrm{kHz}$ dynamic pressure measurements. This technically premixed, swirl stabilized flame exhibited self-excited thermoacoustic oscillations with limit-cycle behavior. A helical precessing vortex core (PVC) was detected within the inner shear layer, between the central recirculation bubble (CRB) and the reactant jets. The PVC was found to be the delineating flow feature for combustion dynamics even at elevated pressure. Sparse dynamic mode decomposition (DMD) of the velocity fields deconvolved the dynamics into a thermoacoustic and PVC mode. The precession of the PVC was at a non-harmonic frequency to the thermoacoustic oscillations, and at least twice that of findings at atmospheric conditions. Nevertheless, the continuous and persistent structure of the PVC allows it promote unsteady heat release to sustain the thermoacoustic cycle. The three dimensional structure of the reactant jets, central recirculation bubble, and PVC was reconstructed by double phase conditioning the reconstructed velocity field. The surface of the CRB was observed to transition between asymmetric and symmetric states depending on the thermoacoustic phase. Analysis of the swirling strength values on the CRB surface indicates the interaction strength between the hydrodynamic structures of the PVC and CRB. When this coupling is large, the heat release determined by the mean $\mathrm{OH}^{*}-\mathrm{CL}$ intensity is maximum. These findings indicate a critical role of the PVC and CRB interaction on combustion in unstable swirl flames at conditions closer to those found in a modern gas turbine engine.
\end{abstract}

Keywords: combustion dynamics, turbulent swirl flame, high pressure, gas turbine, laser diagnostics

\footnotetext{
${ }^{*}$ Corresponding author

Email address: cslabau@purdue.edu (Carson D. Slabaugh )
} 


\section{Introduction}

Swirling flows are often used in aerospace and industrial combustion systems. A bulk swirling flow is typically generated by fixed vanes upstream of the combustion zone. If the geometry of the combustor promotes a sufficient level of swirl, the bulk swirling flow breaks down and generates a central recirculation bubble (CRB) [1]. This allows for hot combusted gases to recirculate back to the fresh reactants, thus stabilizing the flame. A CRB may also be formed by other means (such as a bluff-body), but swirl induces greater mixing through strong shear layers. Swirl also produces a compact flame, because fuel and oxidizer mixing is enhanced through vortex rollup events with the CRB [2]. This characteristic is highly desirable for aviation applications where reducing combustor weight and size is vital.

In a global effort to reduce $N O_{x}$ and particulate matter (smoke) emissions, the gas turbine industry has given increased focus to lean combustion system, with (partial) premixing [3]. An undesirable consequence of this operating condition is an increased susceptibility for combustion dynamics, where pressure oscillations couple with the flame heat release in a positive feedback loop that can negatively impact engine performance and significantly reduce combustor life. Operating near the lean blowout limit allows for smaller hydrodynamic pertubations to greatly affect heat release through extinction and re-ignition events $[4,5]$. Modern combustors also use less liner cooling air to allow more air into the combustion zone and improve the temperature distribution to the turbine. This cooling flow is then less effective at absorbing acoustic oscillations as it begins to act as a hard instead of a porous wall $[6,7]$. The susceptibility of a combustor to instability would ideally be foreseen in the design phase and remedied, but computationally inexpensive numerical tools have not demonstrated such predictive capabilities with engine-relevant conditions and geometries. Extensive studies involving complex computational simulations and experimental measurements have been undertaken to understand and control combustion dynamics. However, more work is required to achieve this capability, especially in practical applications with increased flame power density and complex combustor geometries [8].

As a target case for the International Workshop on Measurement and Computation of Turbulent Flames, the PRECCINSTA gas turbine model combustor (GTMC) has been the subject of extensive experimental and numerical investigation at atmospheric pressure. In a distinct range of operation, this GTMC has been shown to exhibit strong self-excited thermoacoustic pulsations. Meier et al. performed a detailed experimental characterization of this unstable operation by studying two different equivalence ratio conditions [9]. Utilizing multiple laser diagnostics, significant differences were observed between the quiet and noisy (unstable) flames with respect to the turbulent flow structure, mixing characteristics, flame shape, and the reaction progress. These results showed that the feedback loop for the sustained, self-excitation of thermoacoustic oscillations in the PRECCINSTA GTMC (at atmospheric pressure) was an oscillation in mixture fraction coupled with a convective time delay. Franzelli et al. performed LES computations, which agreed with the previous experimental findings of equivalence ratio oscillations driving the thermoacoustic instability $[10]$.

Subsequent investigations with $10 \mathrm{kHz}$ PIV and OH-PLIF identified the presence of a 
helical precessing vortex core (PVC) only in the unstable flame by inspecting the timeevolution of the flow field and proper orthogonal decomposition (POD). The stable flame only displayed periodic formation and ejection of symmetric vortex pairs in the inner shear layer. The PVC was hypothesized to be strongly coupled to the thermoacoustic oscillation, because the PVC precession frequency occurred at an integer multiple of the thermoacoustics $[11,12]$. However, later experimental findings have shown that the PVC frequency can be at non-integer multiples of the thermoacoustic pulsation [13]. Oberleithner et al. identified operating conditions that displayed intermittent flame shape transitions between $\mathrm{V}$ and $\mathrm{M}$ flames in the PRECCINSTA GTMC [14]. It was found that the formation of a PVC was a precursor to the $\mathrm{M}$ flame shape and increased pressure oscillations. The generation of a PVC was coupled to the density and mean flow fields at the combustor inlet. A suppression of the radial density gradient resulted in the formation of the PVC, thus leading to lift-off of the flame root from the combustor nozzle.

Flames must also be studied at industrial engine relevant conditions, because coupling mechanisms can develop from a much broader spectrum of physical processes at high power density and Reynolds number. Swirl stabilized flames are particularly complex because the flow structure supports a wide range of interacting hydrodynamic structures. Experimental studies on the role of a PVC in relation to combustion dynamics are far fewer at elevated pressure and temperatures conditions where industrial engines operate. This is attributed to the increased infrastructure requirements as only a few research labs in the world have capabilities to operate combustors at the megawatt level [15-18]. Combined with the requirements of optical access for laser diagnostics, the sparsity of such investigations in the literature is perhaps unsurprising. The current work adds to the literature on combustion instability at conditions approaching those found in a gas turbine engine.

Slabaugh et al. investigated the PRECCINSTA GTMC at DLR-Stuttgart with simultaneous $\mathrm{OH}^{*}-\mathrm{CL}$, SPIV, and OH-PLIF at two equivalence ratios and at elevated pressure and temperature [13]. They found that the lower equivalence ratio condition led to combustion dynamics and coincided with the appearance of a PVC. At atmospheric conditions, the PVC precession occurred at a constant Strouhal number, and this was still the case for the elevated pressure results. However, the PVC precession frequency was twice the highest values reported for atmospheric operating conditions. A reconstructed velocity field from the ten most energetic POD modes displayed the PVC drastically distorting the CRB. Therefore, the PVC is coupled to the thermoacoustics by modulating mixing in the reacting inner shear layer similar to findings at atmospheric conditions. This study expands upon the previous elevated pressure findings where the time-scale of the PVC precession is nearly four times that of the thermoacoustic instability. An extensive characterization of the three dimensional structure of the PVC and CRB interaction is presented to further elucidate the mechanism of the self-sustained thermoacoustic oscillation. 


\section{Experiment Configuration}

\subsection{Burner and Flame}

With an extensive experimental and numerical database in place for atmospheric pressure flame conditions, the focus of the present work is to characterize the PRECCINSTA GTMC at elevated thermal power density operation. The geometry of the mixer and nozzle have remained unchanged, with natural gas and air being technically premixed (not perfectly premixed [19]) in the swirler section using 12 radial vanes and 12 corresponding $1 \mathrm{~mm}$ fuel injection ports. The exit of the nozzle is $27.85 \mathrm{~mm}$ in diameter, with a rounded, conical center-body aligned to the central axis.

Figure 1 displays the installation of the PRECCINSTA burner into the High Pressure Optical (HIPOT) test rig operated at DLR-Stuttgart. Full optical access to the flame is provided through all four walls of the combustion chamber using a series of fused quartz windows. In-depth descriptions of the combustor geometry and experimental platform is provided in [13]. All operating conditions, including pressures, temperatures, and air/fuel/water mass flow rates were monitored and recorded at a $1 \mathrm{~Hz}$ interval. Dynamic pressures were also measured in the upstream plenum, the combustion chamber, and the downstream section with high-frequency piezo-resistive pressure transducers (Kistler 4043A with a 4603A amplifier). The signals were simultaneously recorded (with dedicated A/D converters) at $100 \mathrm{kHz}$, below the natural frequency of the instruments, along with the intensifier gate signal from the $\mathrm{OH}^{*}$-CL detection system in order to synchronize the optical measurements to the measured acoustic signals.

[Figure 1 about here.]

Previous work investigated two operating conditions: one flame was stable (Flame A) and the other (Flame B) exhibited thermoacoustic instabilities. All conditions were identical between the two cases except for the fuel mass flow rate, which was lower for the unstable case [13]. The presence of a PVC was evident in the flow field measurements from Flame B. The PVC was not present in the stable case, Flame A. Therefore, Flame B was chosen for more extensive analysis relating to the observed thermoacoustics in this work. The global equivalence ratio was 0.58 , and the flame thermal power was $150 \mathrm{~kW}$. The inlet air mass flow rate of $88 \mathrm{~g} / \mathrm{s}$ was preheated to $600 \mathrm{~K}$, and the combustor was operated at $0.5 \mathrm{MPa}$. This resulted in a bulk velocity of $55 \mathrm{~m} / \mathrm{s}$, and a Reynolds number, based on the nonreacting flow, of 130,000. The Damköhler number was estimated to be 0.04 for this flame. This was based on the integral time scale computed in the previous study, and a chemical time scale calculated using the GRI-Mech 3.0 for a laminar flame at identical operating conditions of this work $[13,20]$. The Strouhal number, $S t=f_{P V C} d_{n o z z l e} / u_{x, b u l k}$, of the PVC precession was calculated to be 0.87 , which closely matched previous investigations of the burner at atmospheric conditions [13]. The experiment was always given a minimum of 10 minutes to stabilize, prior to acquiring measurements. $5 \mathrm{~g} / \mathrm{s}$ of unheated inlet air was diverted through the particle seeding system, then reintroduced to the inlet plenum. Though the particle seeding was limited to only short bursts during data acquisition, this unheated flow was 
maintained throughout the entire test by diverting it through a bypass circuit. This process ensured negligible impact on the combustor inlet conditions during data acquisition.

\subsection{Measurement Systems}

Simultaneous measurements of scalar and three-component velocity fields were acquired at a $6 \mathrm{kHz}$ interrogation frequency using combined $\mathrm{OH}^{*}$ chemiluminescence $\left(\mathrm{OH}^{*}-\mathrm{CL}\right)$ and stereoscopic particle image velocimetry (SPIV). A schematic representation of the optical measurement system configuration is given in Figure 2. The reader is referred to [13] for complete details of each sub-system including resolution considerations. The SPIV and $\mathrm{OH}^{*}$-CL cameras had sufficient on-board memory to acquire 5457 double-frame image pairs and 6826 images per run, respectively. Image system calibrations were performed by imaging a dual-sided, dual-plane dot target (LaVision Type 07). The same target was used for the SPIV and $\mathrm{OH}^{*}$-CL systems, hence spatial alignment of all measurement fields was achieved by mapping to the same coordinate system.

[Figure 2 about here.]

Image processing was undertaken to increase image quality for the PIV algorithm. The minimum intensity over the previous and future five images is subtracted to remove background noise and decrease glare. A $7 \times 7$ pixel sliding window intensity normalization is implemented to reduce any laser sheet intensity variations and sets all particles to comparable intensities. Particle cross-correlations were performed using the multi-pass adaptive window offset cross-correlation algorithm in the LaVision commercial software (DaVis 8.4.0). SPIV results presented in this work were processed using a final window size of $24 \times 24$ pixels with $50 \%$ overlap. The corresponding window resolution is $1.25 \mathrm{~mm}$ with vector spacing of $0.63 \mathrm{~mm}$. A $3 \times 3$ sliding window median filter was repeatedly applied three times to remove spurious vectors. The median filter removes velocities outside twice the standard deviation of its neighbors. The removed vectors are replaced with an alternative displacement correlation peak if it is suitable [21]. Vectors were also removed if their correlation peak ratio was less than two. The resulting instantaneous velocity fields had empty spaces where spurious vectors were removed. These locations are filled in by a linear interpolation from their neighbors. An anisotropic denoising filter was applied to better match the interpolated vectors to the flow field [22]. The top two-thirds of the velocity field consistently yielded over $90 \%$ first-choice vectors with less than $2 \%$ (removed) spurious vectors. Internal reflections and other noise sources caused a reduction in vector quality near the bottom of the image, so that region was cropped to avoid contamination of the subsequent, quantitative analysis.

Chemiluminescence from electronically-excited hydroxyl $\left(\mathrm{OH}^{*}\right)$ was imaged using a highspeed CMOS camera coupled to an external two-stage intensifier. An intensifier gate width of $25 \mu \mathrm{s}$ was used for all of the acquired datasets. A 305-315 nm interference filter was placed in-front of the objective lens to isolate the $\mathrm{OH}^{*}$-CL emission. The field of view for the $\mathrm{OH}^{*}$-CL system was approximately $65 \times 80 \mathrm{~mm}$ to capture the full spatial extent of the flame zone. White field correction was applied to remove the spatial intensity variations from the detection system [23]. 


\section{Deconvolution of Flow Dynamics}

Reacting turbulent flows are highly complex due to non-linear coupling of chemistry, turbulence, heat transfer, and acoustics through a vast range of spatiotemporal scales. The interest here lies in the interaction between hydrodynamics and acoustics in a combustor exhibiting sizable pressure oscillations. The amplitude of the thermoacoustics are affected by the hydrodynamics that drive mixing thus reaction rate and heat release. However, these dynamics are perturbed by turbulence in a seemingly stochastic manner. Our method to isolate the large-scale dynamics from the velocity field data is presented below.

Dynamic mode decomposition (DMD) is a parameter-free and data-driven method for understanding complex and high-dimensional datasets. It is in the vein of other lower order dynamics identification algorithms such as POD. DMD is an approximation of the Koopman operator, which characterizes a non-linear system through an infinite dimensional linear operator acting on a set of observables. In practice, the observables are typically the directly measured quantities, such as velocity in this case. An eigendecomposition of the approximated Koopman operator results in a series of modes, each with a growth rate and frequency. DMD generates a linear model of the typically underlying non-linear dynamics. Unlike POD, which associates and ranks modes based on energy, DMD modes are associated with a unique frequency [24, 25]. For example, the dynamics associated with the thermoacoustic oscillation frequency in this dataset were observed across multiple POD modes [13].

DMD was first applied to non-reacting jets where it identified the spatial structure and frequency of the jet's vortex shedding $[26,27]$. DMD has since been extended to the analysis of numerous experimental and numerical efforts in understanding the coupling between hydrodynamics and the resulting thermoacoustics [28-33]. Variants of DMD have been proposed to tackle specific problems such as resolving transient and quasi-periodic dynamics [34]. Methods also exist for debiasing the DMD eigenvalues if the dataset is rank truncated by the most energetic POD modes [35, 36]. The following analysis uses the Exact DMD formulation because of its superior generality [37]. The data does not need to be uniformly sampled in time. Datasets from repeated experiments can be appended to improve accuracy of the modes and frequencies. A brief outline of the DMD implementation is as follows.

The snapshot arrays are formed by flattening the three component, $m \times n$ velocity field at each time step to form $z_{t}$ as

$$
u_{x}, u_{y}, u_{z} \in \mathbb{R}^{m n} \quad z_{t}=\left[\begin{array}{l}
u_{x}(t) \\
u_{y}(t) \\
u_{z}(t)
\end{array}\right] .
$$

Ordering of the velocity components in $z_{t}$ is inconsequential as long as the mapping back to the original data structure, $\mathbb{R}^{m \times n}$, is preserved. The data vectors are then concatenated 
into $X$ and $Y$ arrays as

$$
\begin{aligned}
& X \triangleq\left[\begin{array}{cccc}
\mid & \mid & & \mid \\
z_{0} & z_{1} & \ldots & z_{T-1} \\
\mid & \mid & & \mid
\end{array}\right], \\
& Y \triangleq\left[\begin{array}{cccc}
\mid & \mid & & \mid \\
z_{1} & z_{2} & \ldots & z_{T} \\
\mid & \mid & & \mid
\end{array}\right] .
\end{aligned}
$$

The first time step, $z_{0}$, is solely within $X$. Likewise the last time step, $z_{T}$, is only contained in $Y$. A singular value decomposition (SVD) is performed on the $X$ data array, resulting in

$$
X=U \Sigma V^{*}
$$

The approximated Koopman operator, $\tilde{A}$, is formed as

$$
\tilde{A} \triangleq U^{*} Y V \Sigma^{-1} \text {. }
$$

This is a matrix multiplication of the previously calculated SVD matrices and the $Y$ data array. Next, an eigendecomposition of the square matrix $\tilde{A}$ results in eigenvectors and eigenvalues, $W$ and $\lambda$ respectively. Finally, the dynamic modes of the dataset are then defined as

$$
\Phi \triangleq Y V \Sigma^{-1} W
$$

incorporating the eigenvectors of $\tilde{A}$. The corresponding temporal frequencies (in $\mathrm{Hz}$ ) for each dynamic mode are the imaginary portion of the scaled eigenvalues

$$
f_{i}=\frac{\operatorname{Im}\left\{\ln \left(\lambda_{i}\right)\right\}}{2 \pi \Delta t}
$$

where $\Delta t$ is the measurement sampling frequency.

In order to select the suitable DMD modes for construction of a lower order model, the importance of each mode to the original dynamics is needed. Sparse DMD is chosen as the mode selection method for this study, and the following formulation closely follows the original work of Jovanovic et al. [38]. It has been applied to PIV in non-reacting flows to identify coherent structures and their frequencies. [39, 40]

A complex amplitude is associated with each DMD mode, which dictates its contribution to the dynamics of the lower order model. The vector of optimal DMD mode amplitude is defined as

$$
\alpha=\left[\left(W^{*} W\right) \odot\left(\overline{V_{\text {and }} V_{\text {and }}^{*}}\right)\right]^{-1} \overline{\operatorname{diag}\left(V_{\text {and }} X^{\dagger *} W\right)},
$$

where $*$ denotes the complex conjugate transpose, $\odot$ is a element-wise multiplication, $\bar{D}$ is the complex conjugate of matrix $D, D^{-1}$ is the matrix inverse of $D$, $\operatorname{diag}(\mathrm{D})$ forms a vector 
from the diagonal elements of matrix D, and

$$
V_{a n d}=\left[\begin{array}{ccccc}
1 & \lambda_{0} & \lambda_{0}^{2} & \ldots & \lambda_{0}^{T} \\
1 & \lambda_{1} & \lambda_{1}^{2} & \ldots & \lambda_{1}^{T} \\
\ldots & \ldots & \ldots & \ldots & \ldots \\
1 & \lambda_{n} & \lambda_{n}^{2} & \ldots & \lambda_{n}^{T}
\end{array}\right]
$$

for an $n-1$ and $T-1$ number of DMD modes and time steps, respectively. Each row of the Vandermonde matrix in equation 7 corresponds to a damped or undamped sinusoid governing the temporal evolution of each DMD spatial mode, $\Phi$. Henceforth, these rows are referred to as a temporal mode.

Equation 6 was modified from Jovanovic et al. [38] to use the original snapshot array, $X^{\dagger} \triangleq X \cup Y$ (neither the first nor last time step is missing), necessary for Exact DMD. We seek to find an $\alpha$ that minimizes the reconstruction error defined as the Frobenius norm of the difference between the original snapshots and the dynamics from a sparse set of DMD modes

$$
J(\alpha) \triangleq\left\|X^{\dagger}-\Phi D_{\alpha} V_{a n d}\right\|_{F}^{2},
$$

where $D_{\alpha}$ is a diagonal matrix of the previously calculated optimal mode amplitudes.

Applying a penalty to the cardinality of $\alpha$ would reflect the goal of finding sparse modes, but Jovanovic et al. [38] states that the objective function then becomes intractable for large datasets. Therefore, the objective function is defined as

$$
\min _{\alpha} J(\alpha)+\gamma \sum_{i=1}^{T-1}\left|\alpha_{i}\right|,
$$

where $\gamma$ is the penalty multiplier to the sum of absolute values of the optimal mode amplitudes.

A wide range of sparsity multipliers were initially swept to gauge the DMD mode spectrum for the velocity dataset. However, just two sparsity levels are presented to illustrate the robustness of the modes selected for analysis. Figure 3a shows the eigenvalues of the DMD modes on the complex plane with the unit circle depicted as the dotted yellow line. The non-sparse modes (shown by black o symbols) display increased density near the unit circle. Likewise the sparse DMD modes (red and teal $\times$ ) are only present near the unit circle. Hence sparse DMD preferentially selects neutrally stable dynamics representing limit-cycle behavior, thereby removing modes representing rapidly decaying turbulent fluctuations.

The amplitude spectrum is shown in Figure $3 \mathrm{~b}$ for sparse and non-sparse modes. Identifying the most important modes in the non-sparse spectrum (shown by the black o symbols) is difficult. The highest amplitude modes have large decay rates, thus their influence on the reduced order model only lasts a short period of time. Red and teal $\star$ symbols represent sparse DMD modes with $\gamma$ values of 5000 and 10,000 respectively. Doubling the penalty multiplier from 5000 to 10,000 preserves a few sub $50 \mathrm{~Hz}$ modes along with the 455, 1600, and $1704 \mathrm{~Hz}$ modes. 
Previous POD analysis of the flame revealed the presence of a PVC. The power spectra of these POD temporal modes displayed broadband content centered at $1710 \mathrm{~Hz}$ along with a narrow peak around $450 \mathrm{~Hz}$. The thermoacoustic frequency was determined to be $450 \mathrm{~Hz}$ from analysis of the $\mathrm{OH}^{*}-\mathrm{CL}$ and dynamic chamber pressure [13]. Therefore, the $455 \mathrm{~Hz}$ DMD mode corresponds to the thermoacoustics, and the 1600 and $1704 \mathrm{~Hz}$ modes relate to the PVC.

The DMD mode at $1704 \mathrm{~Hz}$ had greater spatial coherence and more easily identifiable centers of rotation compared to the $1600 \mathrm{~Hz}$ mode. This leads to setting the sparsity structure to only the 455 and $1704 \mathrm{~Hz}$ modes. Figure 4 shows the real part of the selected DMD modes for brevity. The $455 \mathrm{~Hz}$ mode clearly represents a modulation of the reactant jets and CRB. The pulsating inflow, at the same frequency as the combustor and plenum pressure oscillations, plays a key role in the feedback mechanism resulting in combustion dynamics. Therefore, the $455 \mathrm{~Hz}$ DMD mode is referred to as the thermoacoustic mode. Contrastingly, the $1704 \mathrm{~Hz}$ mode displays two adjacent counter-rotating vortices corresponding to the PVC dynamics.

[Figure 3 about here.]

[Figure 4 about here.]

The time evolution of a simplified flow field from these two DMD modes can now be generated. The optimal amplitudes are recalculated by equation 6 for a sparsity structure of just the 455 and $1704 \mathrm{~Hz}$ modes associated with the most prominent limit-cycle dynamics. A superposition of the two DMD modes and the mean velocity field by

$$
\left[\begin{array}{l}
u_{x} \\
u_{y} \\
u_{z}
\end{array}\right]_{\text {mean }}+\left[\Phi D_{\alpha} V_{a n d}\right]_{455+1704}
$$

produces the reconstructed dynamics for further analysis.

Three probe locations in the flow field are selected to demonstrate the effectiveness of the DMD reconstruction in terms of capturing the cyclic oscillations of the underlying data. Figure 5 displays the probe points overlaid on the time averaged velocity field. Points labeled P1-3 are located within the reactant jet, the inner shear layer, and CRB respectively. These probe locations coincide with the location of flow structures found in previous studies of the combustor [12]. The line plots in figure 5 show the axial velocity for the DMD reconstruction and original dataset over last 200 time steps demonstrating reconstruction accuracy even towards the end of the time sequence. The global trends have been preserved, but the turbulent, aperiodic fluctuations are removed by the reconstructed signal similarly to other work using DMD [41]. The superposition of 1704 and $455 \mathrm{~Hz}$ oscillations fits the original data well for P1 and P2. The reconstruction at P3 is dominated by the $455 \mathrm{~Hz}$ mode, because the PVC mode does not strongly perturb the inner most part of the CRB. Overall, the DMD reconstruction acts as a bandpass filter over the original data removing the turbulent fluctuations in each of the probe locations, while leaving the high-power dynamics associated with the large-scale structures of interest. 
[Figure 5 about here.]

\section{Phase-Conditioned Statistics}

Both 455 and $1704 \mathrm{~Hz}$ temporal modes are undamped but stable, and this behavior is previously shown by the time series in figure 5. Conditioning simultaneously acquired datasets on the phase of the temporal mode isolates it from other processes, such as turbulent fluctuations. The instantaneous phase for each DMD temporal mode is calculated by taking the argument of the analytic signal derived from a Hilbert transform of the temporal mode $[42]$.

\subsection{Thermoacoustic Mode}

The chamber dynamic pressure and $\mathrm{OH}^{*}$-CL were phase conditioned by the $455 \mathrm{~Hz} \mathrm{DMD}$ temporal mode representing the thermoacoustic oscillation. The chamber dynamic pressure is band-passed between 450 and $460 \mathrm{~Hz}$ with a zero phase shift 2nd order Butterworth filter before phase conditioning. Each measurement is then sorted into 12 bins resulting in a bin width of $\pm 15^{\circ}$ or $\pm \pi / 12$. Figure 6 shows the phase averages of the $\mathrm{OH}^{*}$-CL images at four equally spaced phase angles. A clear dependence of $\mathrm{OH}^{*}$-CL intensity on the $455 \mathrm{~Hz}$ DMD phase signal is observed, which is consistent with the frequency identified in previous analysis of this flame [13]. Intensity throughout the image nearly doubles from $\pi / 2$ to $3 \pi / 2$. The flame shortens axially and shows a slight radial contraction at its base during the minimum intensity. This reinforces the notion that the $455 \mathrm{~Hz}$ DMD mode corresponds to the thermoacoustic oscillations. The mean intensity is calculated over the spatial domain for each phase angle to better quantify the $\mathrm{OH}^{*}$-CL signal. Figure 7 displays this along with the phase averaged chamber dynamic pressure. A phase lag of $2 \pi / 3$ between the two signals is present. Similar lags have been reported in studies of atmospheric swirl flames. Lower pressure oscillations corresponded to greater phase lag than conditions with higher pressure oscillations in accordance to Rayleigh's criterion [43].

[Figure 6 about here.]

[Figure 7 about here.]

\subsection{D Structure}

Swirl stabilized flames result in highly three-dimensional flow fields, so it is helpful to illustrate the full dimensionality of the most influential flow structures. Two-dimensional representations can be ambiguous. For example, uncertainty in the origin of isolated flame kernels was investigated in the PRECCINSTA burner at atmospheric conditions. Further analysis pinned down the flame kernels to convection from the out-of-plane PVC motion and not auto-ignition [44]. High repetition rate measurements allow for phase locking multiple dynamic processes within one dataset. In contrast to much earlier work where low speed 
diagnostics were locked to a plenum pressure measurement, and thus were only able to resolve trends with the respect to the frequency locked process [19].

PVCs have been identified in swirling flows by phase averages of 2D SPIV data using the harmonic content of POD temporal coefficients as the phase reference in multiple previous studies. Stohr et al. reconstructed multiple coherent vortex cores from the DLR dual-swirl burner with no thermoacoustic oscillations $[45,46]$. Steinberg et al. performed a doubly-phase-resolved analysis on the dual-swirl burner at operating conditions exhibiting thermoacoustic oscillations [43]. The plenum pressure was used to determine the thermoacoustic phase, and the POD temporal coefficient from one mode was used for the PVC phase. The geometry and thermoacoustic coupling between the PVC and flame surface areas was investigated for three different flames. Azimuthal symmetry of the PVC was assumed in all of these studies.

Similar to previous work, the 3D structure of the PVC coupled with thermoacoustic oscillations is generated by a doubly phase conditioned analysis. The phase signal of the $1704 \mathrm{~Hz}$ DMD temporal mode is calculated similarly to the $455 \mathrm{~Hz}$ phase signal used for conditioning from the previous section. This corresponds to the phase angle (or angular orientation) of the PVC, which exists throughout the thermoacoustic cycle. The DMD reconstructed velocity field is then phase conditioned by both 455 and $1704 \mathrm{~Hz}$ DMD phase signals. The PVC and thermoacoustics are separated into 16 and 12 bins respectively. This results in 192 phase bins with roughly 28 velocity fields each that are then averaged. The average velocity field for each phase bin was found to have narrow $3 \sigma$ confidence intervals leading to the conclusion that the statistic is sufficiently resolved. Further details on the bin number selection is provided in Appendix A. The azimuthal dimension is generated by rotating the PVC phase averaged velocity around the $Y=0$ axis at regular intervals of $22.5^{\circ}$. The velocity fields are cropped below $Y=0$, so the rotation does not produce any interference at $\pi$ intervals. The azimuthal location of the $0^{\circ} \mathrm{PVC}$ phase is arbitrary, because the thermoacoustic phase has no phase relationship to the PVC orientation.

Figure 8 shows three coherent flow structures at all 12 thermoacoustic phase angles. The PVC is defined by an iso-surface at a swirling strength $\left(\lambda_{c i}\right)$ of $8000 / s$. This is a user specified threshold chosen to produce the clearest reconstruction. Swirling strength has been used by numerous groups for vortex identification [45-48] and is defined as the imaginary portion of the complex eigenvalues of the velocity gradient tensor. It is Galilean invariant and ignores shear layers which have large vorticity magnitudes but no spiraling motion. Also swirling strength is extendable to compressible flows, which is not the case for other popular methods such as Q and $\lambda_{2}$ criteria [49]. Nevertheless, the authors would like to note that the visualizations with $\mathrm{Q}$ criteria does produce similar results as $\lambda_{c i}$. Iso-surfaces of $u_{x}=0 \mathrm{~m} / \mathrm{s}$ and $u_{x}=80 \mathrm{~m} / \mathrm{s}$ represent the boundary of the CRB and a swirling reactant jet, respectively. The $u_{x}=80 \mathrm{~m} / \mathrm{s}$ threshold is empirically determined by the axial velocity magnitude that encompasses most of the reactant jet in the mean velocity field. Contours of $u_{x}=0 \mathrm{~m} / \mathrm{s}$ have been used to demarcate the CRZ in previous studies on GTMCs, so this work follows that convention [13, 43, 45].

The minimum axial extent of the PVC occurs at $\theta_{T A}=\pi / 3$ and grows into a coherent helical shape by $\theta_{T A}=\pi$. The PVC is bound by the CRB and jet in-flow throughout the 
measurement domain. This is clearly shown from $\theta_{T A}=\pi$ to $3 \pi / 2$, and alludes to the role of the PVC in transporting hot gases from the CRB into the reactant jets to sustain combustion. The CRB is highly asymmetric and contracted at the base from $\theta_{T A}=0$ to $\pi / 3$ when the reactant jets and PVC are weak, thus no longer confining the CRB. However, the jets and PVC then increase in strength and spatial extent, thereby molding the CRB into a more symmetric shape. The CRB undergoes radial expansion beginning at $\theta_{T A}=\pi / 2$, thereby pushing the PVC and jets outward. Eventually the jets rapidly decay near $\theta_{T A}=3 \pi / 2$ and the PVC along with it. The thermoacoustic cycle then restarts with a large CRB unimpeded by a strong reactant jets.

[Figure 8 about here.]

Figure 9 displays a 2D slice of the reconstructed 3D flow structures from figure 8 overlaid on the phase averaged $\mathrm{OH}^{*}-\mathrm{CL}$. The $2 \mathrm{D}$ slice represents a fixed phase angle, thus spatial orientation, for the PVC. $\mathrm{OH}^{*}-\mathrm{CL}$ is a path integrated measurement, so any spatial relationship with the 2D contours is not exact. However, this figure further illustrates that the PVC is spatially confined between the reactant jets and the CRB. As expected for a reacting shear layer, the reactant jets typically border the edge of heat release. The expansion and contraction of the CRB is greater than any change in the $\mathrm{OH}^{*}-\mathrm{CL}$ topology. However, the $\mathrm{OH}^{*}$-CL intensity is highly correlated to size of the CRB. For example, the CRB is shown to be contracted from $\theta_{T A}=0$ to $\pi$ coinciding to the lowest $\mathrm{OH}^{*}-\mathrm{CL}$ intensity distributions.

[Figure 9 about here.]

The evolution of these structures can be more quantitatively described by figure 10 showing the volume enclosed by each iso-surface with respect to the thermoacoustic phase angle. The volume of the PVC and jets closely track each other with a small phase shift around one phase bin spacing. The CRB and jet volumes have a much larger phase shift. The jet volume is maximum at $\theta_{T A}=\pi$, while the CRB grows to a maximum at $\theta_{T A}=11 \pi / 6$. At this phase, the jet volume has decreased dramatically, and the PVC volume is begins to reduce. However, the $\mathrm{CRB}$ is now unconstrained and reaches the maximum volume. The described sequence matches the visualization of figure 8 .

[Figure 10 about here.]

The CRB is a source of hot gas that acts to assist ignition and stabilize the high-power swirl flame. Recirculated combustion products are transported into the reactant jets through the shear layer between the two structures. The volumetric flow rate $(\dot{Q})$ through the CRB surface is calculated at each face of the interpolated zero axial velocity iso-surface by a dot product of velocity and the area vector. $\dot{Q}$ out of the CRB is then equal to the sum over the entire iso-surface, and it is plotted against the thermoacoustic phase angle in figure 11. Values are positive throughout the thermoacoustic cycle indicating a net flow out of the CRB, which is fed by entrainment due to vortex breakdown downstream of the field of view. This is expected, because the role of the CRB is to transport combusted hot gases 
back to the reactant jets to maintain ignition. Variation through the thermoacoustic cycle is similar between the CRB volume and $\dot{Q}$, but $\dot{Q}$ stays near its largest magnitudes even when the CRB volume is reduced from its maximum. This is illustrated from $\theta_{T A}=7 \pi / 6$ to $3 \pi / 2$. This indicates transport out of the CRB is significant even at the phases where the CRB is still undergoing expansion to a maximum. Consequentially, the inflow into the CRB was observed to increase at these phase angles. The lowest $\dot{Q}$ is at $\pi / 2$, which is near the minimum $\mathrm{CRB}$ volume and mean $\mathrm{OH}^{*} \mathrm{CL}$ intensity. The maximum $\dot{Q}$ and $\mathrm{OH}^{*}{ }_{-} \mathrm{CL}$ intensity also coincide at nearly the same thermoacoustic phase. This can be attributed to a few causes. At around $\theta_{T A}=\pi / 2$, the reactant jet volume is increasing but still low. Reduced reactant inflow along with low outflow from the CRB causes the observed minimal heat release. Moreover, the reduced flow of hot gases from the CRB into the reacting shear layer results in less reactant preheating. This phenomena can also cause a decrease in flame speed and reaction rate [50], thereby also resulting in lower heat release at this point in the thermoacoustic cycle.

[Figure 11 about here.]

\section{CRB and PVC Coupling}

The PVC influences combustion by generating flame roll-up events that rapidly mix burned and unburned gas to increase reaction rates as shown by previous studies with simultaneous SPIV and OH-PLIF measurements [45]. Regions of large swirling strength on the CRB surface gives an indication of contact with the PVC, thus greater transport between the recirculated hot product gas and fresh reactants. The CRB definition as the $u_{x}=0 \mathrm{~m} / \mathrm{s}$ surface is physically robust, because that is the interface where recirculated gas with negative $u_{x}$ meets the reactant inflow with positive $u_{x}$. Again, the PVC does not have a strict definition, and a criteria based on visualization was used in figures 8 and 10. Nevertheless, figure 12 shows the CRB surface area with $\lambda_{c i} \geq 8000 / s$, which was the threshold used to identify the PVC previously. This provides a sense of how much of the CRB surface is impacted by intense vortical flow. Figures 14 and 13 illustrate the unthresholded distribution of $\lambda_{c i}$ on the CRB; thereby, reinforcing the quantitative analysis of the interaction between the CRB and PVC. Figure 13 displays the surface area weighted $\mathrm{PDF}$ of $\lambda_{c i}$ on the $\mathrm{CRB}$ for four equally spaced phases through the thermoacoustic cycle. Figure 14 shows the surface of the CRB colored by $\lambda_{c i}$ with translucent lower magnitudes to display the helical structure especially for $\theta_{T A}=\pi / 2$. The view is rotated by approximately $180^{\circ}$ from figure 8 to provide another view of the reconstructed PVC and CRB.

[Figure 12 about here.]

[Figure 13 about here.] 
[Figure 14 about here.]

The PVC and CRB coupling is weakest near $\theta_{T A}=\pi / 2$. The minimum area of $\lambda_{c i}$ on the CRB shown in figure 12 occurs at $\theta_{T A}=\pi / 3$ and corresponds to the minimum PVC volume in figure 10. The CRB is observed to be heavily contracted and the PVC just starting to grow at $\theta_{T A}=\pi / 2$ in figure $14 \mathrm{~b}$. This is also when the mean $\mathrm{OH}^{*}$-CL intensity figure 7) and CRB $\dot{Q}$ (figure 11) are nearing or at their minimums. This suggests that when the PVC is weakly interacting with the CRB, then transport of hot gas out of the CRB decreases and heat release is, correspondingly, reduced.

Moving to $\theta_{T A}=\pi$ and $3 \pi / 2$, the peak in the distribution at zero swirling strength (figure 13 ) is less prominent. A more uniform distribution also appears from $\lambda_{c i}=2000 / \mathrm{s}$ to $9000 / \mathrm{s}$; a sign that the rotation within the PVC has increased. Values of $\lambda_{c i} \geq 12,000 / \mathrm{s}$ are most abundant at $\theta_{T A}=3 \pi / 2$. The PVC enhances mixing but could also produce significant local quenching at the operating condition investigated in this work. Findings in the DLR dualswirl burner (DSB) at low Damköhler numbers reported that the PVC induced high enough flame stretch rates to cause local quenching of flame kernels and delaying heat release [51]. The manner of premixing in the DSB is different than the PRECCINSTA burner, so the sensitivity of local quenching to flame stretch rates could be altered. However, the flames of the DSB has been noted to operate in a premixed manner, so the comparison is reasonable [52]. This mechanism could be attributing to lower heat release at $\theta_{T A}=\pi$ than $3 \pi / 2$ because the median $\lambda_{c i}$ at $\theta_{T A}=\pi$ is greater than the median at $3 \pi / 2$. In addition, the surface area of large $\lambda_{c i}$ values on the CRB is much larger at $\theta_{T A}=3 \pi / 2$ than $\pi$. This is demonstrated visually in figure 14c-d by the radial expansion of the CRB volume with the PVC maintaining its helical structure. Therefore, the PVC and CRB intersection and coupling is near maximum at $\theta_{T A}=3 \pi / 2$, which corresponds to the second highest mean $\mathrm{OH}^{*}$-CL intensity.

Starting the cycle again at $\theta_{T A}=0$, the PVC begins to wane as shown by a reduced intersection with the CRB near its root in figure 14a. The peak at $0 \lambda_{c i}$ has reappeared in the distribution of figure 13 , but $\lambda_{c i} \geq 6000 / s$ values are still present in high quantities. These larger $\lambda_{c i}$ values correspond to the presence of the PVC at the top half of the CRB as shown in figure 14a. Looking back to figure 7, this corresponds to an intermediate value in the global heat release indicated by $\mathrm{OH}^{*}$ chemiluminescence.

As the structure of the PVC and CRB is modulated due to the combustor pressure oscillations, mixing between the reactants and recirculated product gas is affected. When the CRB expands radially, its surface area increases. The PVC is constrained between the reactant jets and the $\mathrm{CRB}$ such that the contact between the CRB and PVC is increased. Under these conditions, mixing between the recirculated products and incoming reactants is enhanced, modulating heat release. As the cycle progresses, the CRB, PVC, and reactant jets are reduced in size and strength, thereby reducing the interaction of these structures within the confined volume of the combustor. Less intersection between the CRB and PVC causes a reduction in transport through the reaction zone, continuing the cycle. The dynamics of the PVC are not a direct cause of the instability. The precession frequency of the PVC is $1704 \mathrm{~Hz}$, which does not correspond to the frequency of the thermoacoustic 
instability at $455 \mathrm{~Hz}$ or an under/overtone of that frequency. Nevertheless, the PVC and CRB coupling demonstrates that the presence of the PVC is an important factor in driving and sustaining combustion instability, even at elevated pressure and power conditions when the time-scale of the PVC precession is a factor of 2-3 shorter than in previous investigations of this burner.

\section{Conclusions}

Simultaneous $6 \mathrm{kHz}$ SPIV, OH*-CL, and $100 \mathrm{kHz}$ pressure measurements were acquired in the PRECCINSTA GTMC at elevated pressure and temperature to emulate more enginerealistic conditions. Sparse DMD was performed on the measured velocity fields and successfully identified the thermoacoustic $(455 \mathrm{~Hz})$ and PVC $(1704 \mathrm{~Hz})$ modes as dominant. Both frequencies were similar to those identified in previous work of analysis upon POD results. Reconstructing the velocity time series with the mean velocity and the two DMD modes reasonably matched the temporal evolution to the raw SPIV results. However, the incoherent turbulent fluctuations were removed resulting in velocity field reconstruction that contained only the principal dynamic content. Phase conditioning the $\mathrm{OH}^{*}-\mathrm{CL}$ and dynamic chamber pressure on the thermoacoustic DMD temporal mode resulted in an expected sinusoidal modulation that agreed with previous analysis of this dataset.

A three dimensional reconstruction of the flow field was created by double phase conditioning off the two DMD temporal modes. The reactant jets, PVC, and CRB were tracked throughout the thermoacoustic cycle. The CRB displayed large asymmetric distortions when the PVC and jets were decayed. However, the CRB was molded into a symmetric structure when the PVC and jets gained strength to spatially constrain the CRB. Tying together multiple derived quantities and statistics from the phase conditioned results, it was found that lower levels of heat release generally coincided with reduced interaction between the PVC and CRB. Likewise the thermoacoustic phases with the highest amount of intersection between the PVC and CRB displayed the largest levels of heat release. This was also accompanied by a swelling of the CRB and an increase in volumetric flow rate out of the CRB.

This work has shown that the presence of a PVC between the CRB and the reactant jets remains a key factor in the sustainment of thermoacoustic oscillations at elevated pressure and power for this flame. Despite very different characteristic hydrodynamic time-scales, compared to low power conditions, the rapid mixing induced by the PVC remains a significant mechanism for combustion dynamics in this configuration.

\section{Appendix A: Phase Average Quality}

Confidence interval estimates are used to assess the quality of the 192 phase averages from the double phase conditioning. A three sigma confidence level is calculated for each phase average and each velocity component. This is interpreted as to mean that $99.7 \%$ of repeated experiments contain the true phase average in their confidence intervals. Therefore, 
smaller confidence intervals are desired as a indication of reduced variance. The variability of an average should be zero in the limit of infinite samples.

All three velocity components at probe locations P1-3 corresponding to figure 5 were investigated. However, the widest confidence interval occurred for $u_{x}$, so $u_{y}$ and $u_{z}$ are not shown for brevity. The variation of the confidence interval of $u_{x}$ at P2 is shown in figure 15 for the double phase averages with 192 bins presented in this study. The values represented by the color bar correspond to the half-intervals. For example, the largest confidence interval in figure 15 occurs at $\left(\theta_{T A}=7 \pi / 6, \theta_{P V C}=9 \pi / 8\right)$ and is equal to the mean $\pm 3.27 \mathrm{~m} / \mathrm{s}$. The half-intervals are mainly dependent on $\theta_{T A}$ with two bands of larger widths. $u_{z}$ displays similar patterns but at different $\theta_{T A}$ ranges, while $u_{y}$ shows more dependence on $\theta_{P V C}$.

Table 1 shows the minimum, maximum, and mean half-interval of each probe location for the phase averages. The labels subscripted with $12 \times 16$ denote 192 phase averages used in this work. For contrast, the same confidence interval calculation is performed for double phase averages with the thermoacoustic and PVC phases each split into only 4 bins. In addition, phase averages of the raw dataset are constructed using the instantaneous phase of the $450-460 \mathrm{~Hz}$ band-passed dynamic chamber pressure, and the averages are computed for eight phase bins. No phase conditioning on the PVC dynamics is performed on the raw dataset.

As expected, averaging over more velocity fields produces tighter confidence intervals. The mean half-intervals at every probe point for $4 \times 4$ is less than the $12 \times 16$ case. The minimum half-intervals are larger for the $4 \times 4$ case, because the larger phase bins contain velocity fields with more variance. The reduction in the mean half-interval between the different bin size configurations is small, because the DMD reconstructed velocity fields are purely sinusoidal with no phase modulation. This is not the case for the raw dataset, which has the largest confidence intervals at P1-2 with a comparable interval as the $12 \times 16$ case for P3. More phase bins than the $12 \times 16$ case could have been used with small impact to the confidence intervals, but this was enough for reconstructing of the $3 \mathrm{D}$ structure of the PVC, CRB, and reactant jets through the thermoacoustic cycle.

[Figure 15 about here.]

[Table 1 about here.]

\section{Acknowledgments}

Carson D. Slabaugh acknowledges travel support from the John Zink Institute Fellowship Fund.

\section{References}

[1] O. Lucca-Negro, T. O'Doherty, Vortex breakdown: A review, Prog. Energy Combust. Sci. 27 (2001) 431-481. 
[2] R. H. Chen, J. F. Driscoll, The role of the recirculation vortex in improving fuel-air mixing within swirling flames, Symp. (Int.) Combust. 22 (1989) 531-540.

[3] A. H. Lefebvre, B. R. Ballal, Gas Turbine Combustion, CRC Press, 1998.

[4] Y. Huang, V. Yang, Dynamics and stability of lean-premixed swirl-stabilized combustion, Prog. Energy Combust. Sci. 35 (2009) 293-364.

[5] S. Candel, D. Durox, T. Schuller, J.-F. Bourgouin, J. P. Moeck, Dynamics of swirling flames, Annu. Rev. Fluid Mech. 46 (2014) 147-173.

[6] T. C. Lieuwen, V. Yang, Combustion instabilities in gas turbine engines: operational experience, fundamental mechanisms and modeling, Progress in astronautics and aeronautics, American Institute of Aeronautics and Astronautics, 2005.

[7] G. A. Richards, M. C. Janus, Characterization of Oscillations During Premix Gas Turbine Combustion, J. Eng. Gas Turbine Power 120 (1998) 294-302.

[8] T. Poinsot, Prediction and control of combustion instabilities in real engines, Proc. Combust. Inst. 36 (2017) 1-28.

[9] W. Meier, P. Weigand, X. Duan, R. Giezendannerthoben, Detailed characterization of the dynamics of thermoacoustic pulsations in a lean premixed swirl flame, Combust. Flame 150 (2007) 2-26.

[10] B. Franzelli, E. Riber, L. Y. Gicquel, T. Poinsot, Large Eddy Simulation of combustion instabilities in a lean partially premixed swirled flame, Combust. Flame 159 (2012) 621-637.

[11] W. Meier, I. Boxx, M. Stohr, C. D. Carter, Laser-based investigations in gas turbine model combustors, Exp. Fluids 49 (2010) 865-882.

[12] I. Boxx, C. M. Arndt, C. D. Carter, W. Meier, High-speed laser diagnostics for the study of flame dynamics in a lean premixed gas turbine model combustor, Exp. Fluids 52 (2010) 555-567.

[13] C. D. Slabaugh, I. Boxx, S. Werner, R. P. Lucht, W. Meier, Structure and Dynamics of Premixed Swirl Flames at Elevated Power Density, AIAA J. 54 (2016) 946-961.

[14] K. Oberleithner, M. Stöhr, S. Ho, C. M. Arndt, A. M. Steinberg, Formation and flame-induced suppression of the precessing vortex core in a swirl combustor : Experiments and linear stability analysis, Combust. Flame 162 (2015) 3100-3114.

[15] D. Kroniger, P. Vinnemeier, C. Rudolf, M. Wirsum, High Pressure Combustion Test Rig for 10 MW Full Scale Gas Turbine Combustors, ASME Turbo Expo (2014).

[16] S. E. Meyer, S. D. Heister, C. Slabaugh, R. P. Lucht, A. Pratt, R. M. Gejji, M. Bedard, A. Lemcherfi, Design and Development of the High Pressure Combustion Laboratory at Purdue University, AIAA Propulsion and Energy Forum (2017).

[17] C. D. Slabaugh, A. C. Pratt, R. P. Lucht, S. E. Meyer, M. Benjamin, K. Lyle, M. Kelsey, The development of an optically accessible, high-power combustion test rig, Rev. Sci. Instrum. 85 (2014).

[18] O. Lammel, H. Schütz, G. Schmitz, R. Lückerath, M. Stöhr, B. Noll, M. Aigner, M. Hase, W. Krebs, FLOX® Combustion at High Power Density and High Flame Temperatures, J. Eng. Gas Turbine Power 132 (2010) 121503.

[19] W. Meier, P. Weigand, X. R. Duan, R. Giezendanner-Thoben, Detailed characterization of the dynamics of thermoacoustic pulsations in a lean premixed swirl flame, Combust. Flame 150 (2007) 2-26.

[20] G. P. Smith, D. M. Golden, M. Frenklach, B. Eiteener, M. Goldenberg, C. T. Bowman, R. K. Hanson, S. Song, W. C. Gardiner, V. V. Lissianski, Z. W. Qin, 2015. http://www . me .berkeley .edu/gri_mech/.

[21] J. Westerweel, Efficient detection of spurious vectors in particle image velocimetry data, Exp. Fluids 247 (1994) 236-247.

[22] B. Wieneke, PIV anisotropic denoising using uncertainty quantification, Exp. Fluids 58 (2017) 1-10.

[23] J. S. Lim, Two-dimensional Signal and Image Processing, Prentice-Hall, Inc., Upper Saddle River, NJ, USA, 1990.

[24] I. Mezić, Spectral Properties of Dynamical Systems , Model Reduction and Decompositions, Nonlinear Dyn. 41 (2005) 309-325.

[25] K. K. Chen, J. H. Tu, C. W. Rowley, Variants of dynamic mode decomposition: Boundary condition, Koopman, and fourier analyses, J. Nonlinear Sci. 22 (2012) 887-915.

[26] P. J. Schmid, Dynamic mode decomposition of numerical and experimental data, J. Fluid Mech. 656 
(2010) 5-28.

[27] C. W. Rowley, I. MeziĆ, S. Bagheri, P. Schlatter, D. S. Henningson, Spectral analysis of nonlinear flow, J. Fluid Mech. 641 (2009) 115-127.

[28] S. Roy, J. C. Hua, W. Barnhill, G. H. Gunaratne, J. R. Gord, Deconvolution of reacting-flow dynamics using proper orthogonal and dynamic mode decompositions, Phys. Rev. E Stat. Nonlin. Soft Matter Phys. 91 (2015) 1-16.

[29] T. Buschhagen, R. Gejji, J. Philo, L. Tran, J. Enrique Portillo Bilbao, C. D. Slabaugh, Experimental Investigation of Self-Excited Combustion Instabilities in a Lean, Premixed, Gas Turbine Combustor at High Pressure, J. Eng. Gas Turbine Power 140 (2018) 111503-111509.

[30] T. Sayadi, P. J. Schmid, F. Richecoeur, D. Durox, Parametrized data-driven decomposition for bifurcation analysis, with application to thermo-acoustically unstable systems, Phys. Fluids 27 (2015).

[31] J. P. Moeck, J. F. Bourgouin, D. Durox, T. Schuller, S. Candel, Tomographic reconstruction of heat release rate perturbations induced by helical modes in turbulent swirl flames, Exp. Fluids 54 (2013).

[32] C. Huang, M. Harvazinski, W. Anderson, V. Sankaran, Analysis of Self-Excited Combustion Instability using Decomposition Techniques, AIAA J. 54 (2016) 1-18.

[33] A. Ghani, T. Poinsot, L. Gicquel, J. D. Müller, LES Study of Transverse Acoustic Instabilities in a Swirled Kerosene/Air Combustion Chamber, Flow Turbul. Combust. 96 (2016) 207-226.

[34] C. W. Rowley, S. T. Dawson, Model Reduction for Flow Analysis and Control, Annu. Rev. Fluid Mech. 49 (2017) 387-417.

[35] M. S. Hemati, C. W. Rowley, E. A. Deem, L. N. Cattafesta, De-biasing the dynamic mode decomposition for applied koopman spectral analysis of noisy datasets, Theor. Comput. Fluid Dyn. 31 (2017) 349-368.

[36] S. T. M. Dawson, M. S. Hemati, M. O. Williams, C. W. Rowley, Characterizing and correcting for the effect of sensor noise in the dynamic mode decomposition, Exp. Fluids 57 (2016) 1-19.

[37] J. H. Tu, C. W. Rowley, D. M. Luchtenburg, S. L. Brunton, J. N. Kutz, On Dynamic Mode Decomposition: Theory and Applications, J. Comput. Dyn. (2014) 391-421.

[38] M. R. Jovanović, P. J. Schmid, J. W. Nichols, Sparsity-promoting dynamic mode decomposition, Phys. Fluids 26 (2014) 024103.

[39] A. Ianiro, K. P. Lynch, D. Violato, G. Cardone, F. Scarano, Three-dimensional organization and dynamics of vortices in multichannel swirling jets, J. Fluid Mech. 843 (2018) 180-210.

[40] B. Ponitz, M. Sastuba, C. Brücker, 4D visualization study of a vortex ring life cycle using modal analyses, J. Vis. 19 (2016) 237-259.

[41] P. J. Schmid, D. Violato, F. Scarano, Decomposition of time-resolved tomographic PIV, Exp. Fluids 52 (2012) 1567-1579.

[42] F. Güthe, B. Schuermans, Phase-locking in post-processing for pulsating flames, Meas. Sci. Technol. 18 (2007) 3036.

[43] A. Steinberg, I. Boxx, M. Stöhr, W. Meier, C. Carter, Effects of flow structure dynamics on thermoacoustic instabilities in swirl-stabilized combustion, AIAA J. 50 (2012) 952-967.

[44] I. Boxx, C. D. Carter, M. Stöhr, W. Meier, Study of the mechanisms for flame stabilization in gas turbine model combustors using kHz laser diagnostics, Exp. Fluids 54 (2013) 1532.

[45] M. Stöhr, I. Boxx, C. D. Carter, W. Meier, Experimental study of vortex-flame interaction in a gas turbine model combustor, Combust. Flame 159 (2012) 2636-2649.

[46] M. Stöhr, I. Boxx, C. Carter, W. Meier, Dynamics of lean blowout of a swirl-stabilized flame in a gas turbine model combustor, Proc. Combust. Inst. 33 (2011) 2953-2960.

[47] R. J. Adrian, K. T. Christensen, Z.-C. Liu, Analysis and interpretation of instantaneous turbulent velocity fields, Exp. Fluids 29 (2000) 275-290.

[48] J. Zhou, R. J. Adrian, S. Balachandar, T. M. Kendall, Mechanisms for generating coherent packets of hairpin vortices in channel flow, J. Fluid Mech. 387 (1999) 353-396.

[49] V. Kolár, Compressibility Effect in Vortex Identification, AIAA J. 47 (2009) 473-475.

[50] Y. Wu, V. Modica, B. Rossow, F. Grisch, Effects of pressure and preheating temperature on the laminar flame speed of methane/air and acetone/air mixtures, Fuel 185 (2016) $577-588$. 
[51] M. Stöhr, C. M. Arndt, W. Meier, Effects of Damköhler number on vortex-flame interaction in a gas turbine model combustor, Proc. Combust. Inst. 34 (2013) 3107-3115.

[52] P. Weigand, W. Meier, X. R. Duan, W. Stricker, M. Aigner, Investigations of swirl flames in a gas turbine model combustor: I. Flow field, structures, temperature, and species distributions, Combust. Flame 144 (2006) 205-224. 


\section{List of Figures}

1 Schematic of test article . . . . . . . . . . . . . . . . . . . . 21

2 Schematic of measurement system . . . . . . . . . . . . . . . . . . . . 22

3 Argand diagram of DMD eigenvalues . . . . . . . . . . . . . . . . . 23

4 Selected DMD Spatial Modes . . . . . . . . . . . . . . . . . 24

5 DMD reconstruction comparison . . . . . . . . . . . . . . . 25

6 Phase Averaged $\mathrm{OH}^{*} \mathrm{CL} \ldots \ldots \ldots \ldots$. . . . . . . . . . . . . 26

$7 \quad$ Phase Average of Pressure and Mean OH*-CL . . . . . . . . . . . . . . . . . 27

8 Double Phase Averaged Reconstruction . . . . . . . . . . . . . . . . . . 28

9 Double Phase Averaged Reconstruction with $\mathrm{OH}^{*}$-CL . . . . . . . . . . . . . 29

10 Volume of reactant jets, CRB, and PVC . . . . . . . . . . . . . 30

11 Flux out of the CRB . . . . . . . . . . . . . . . . . . . 31

12 CRB surface area with high swirling strength . . . . . . . . . . . . . . 32

13 PDF of swirling strength on the CRB surface . . . . . . . . . . . . . 33

14 Surface of the PVC and CRB colored by swirling strength . . . . . . . . . 34

15 Confidence half intervals . . . . . . . . . . . . . . . . . . . 35 


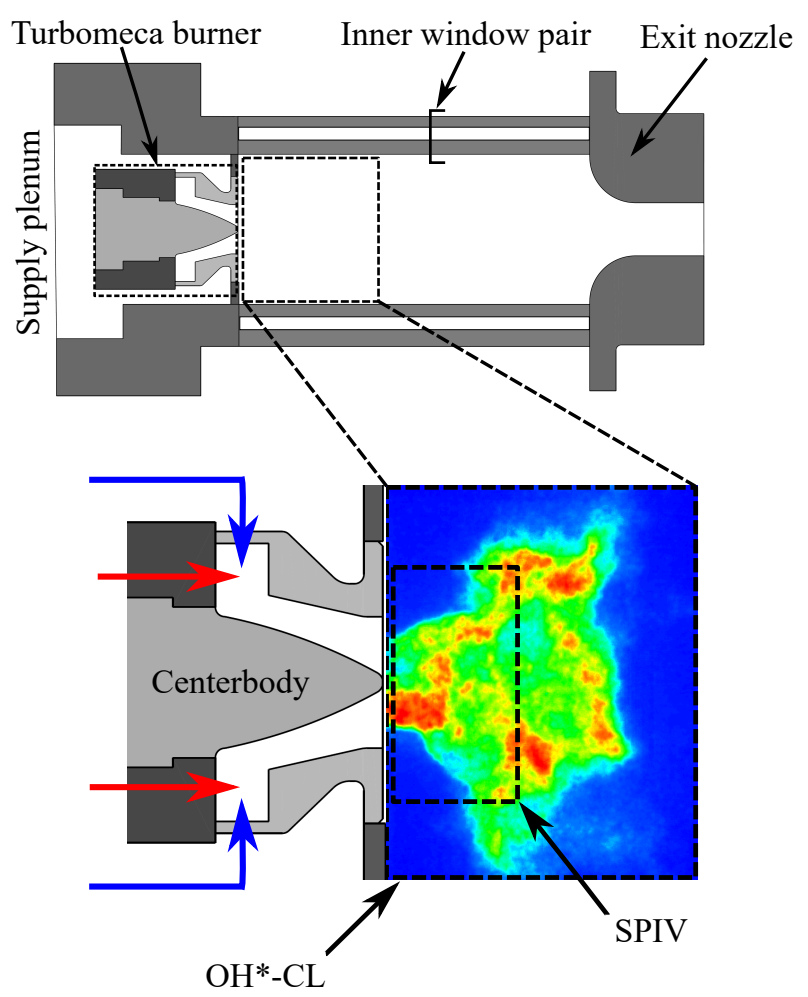

Figure 1: Schematic of PRECCINSTA burner installed into HIPOT test rig with measurement fields of view indicated. 


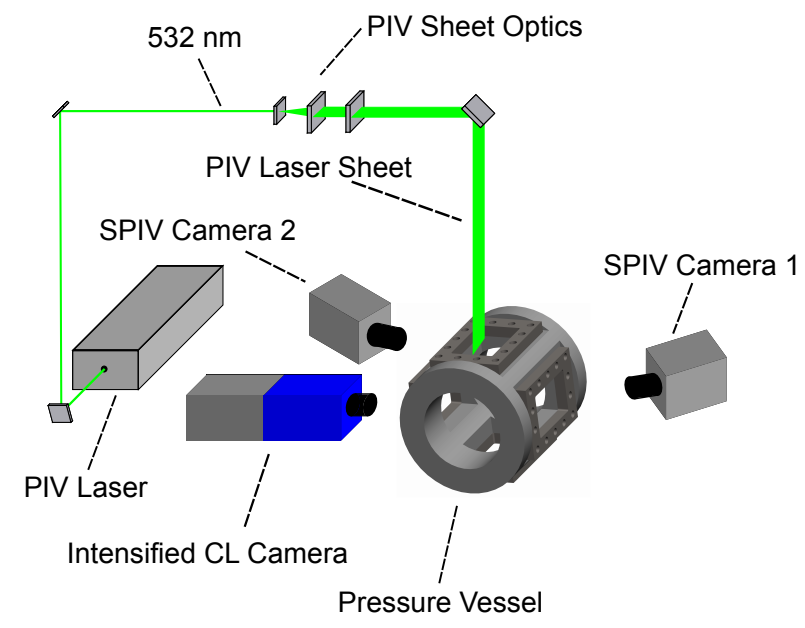

Figure 2: Schematic of measurement system. 

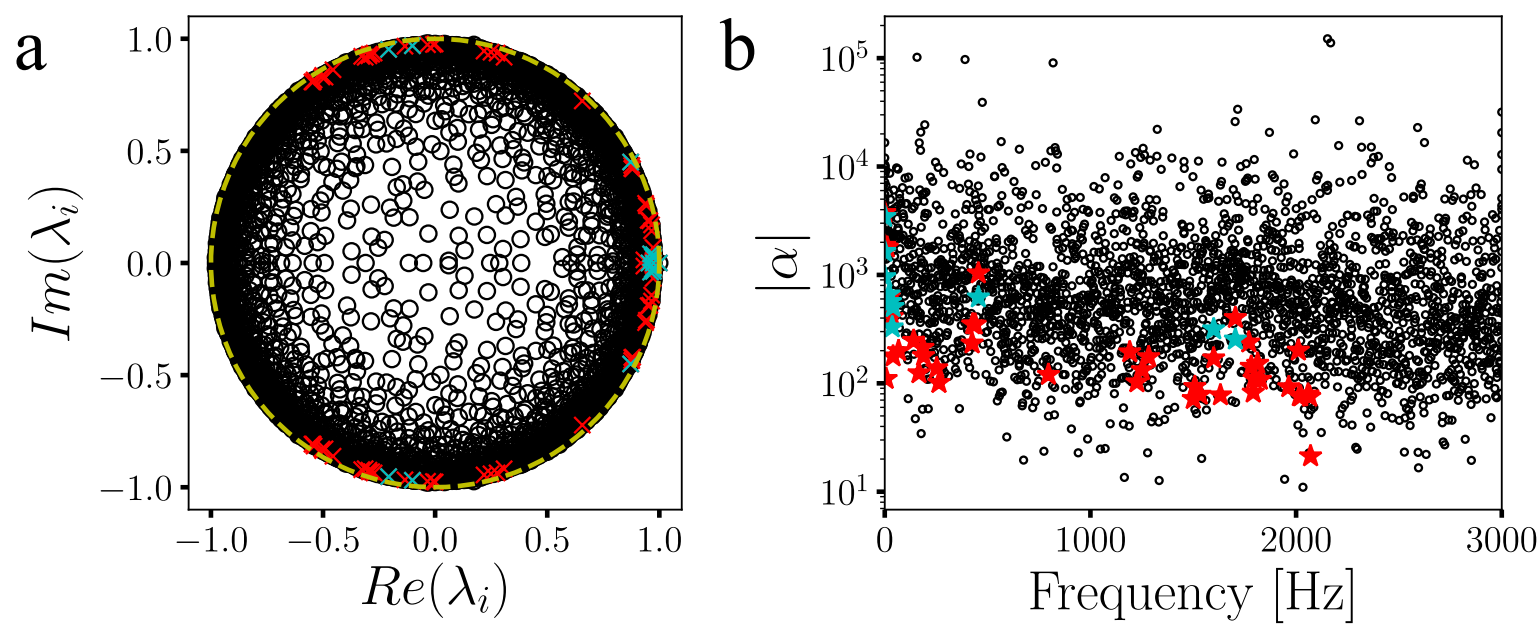

Figure 3: Argand diagram of DMD eigenvalues (a) and amplitude spectra (b) for sparse and non-sparse results. Red and teal symbols represent $\gamma$ values of 5,000 and 10,000 respectively. 

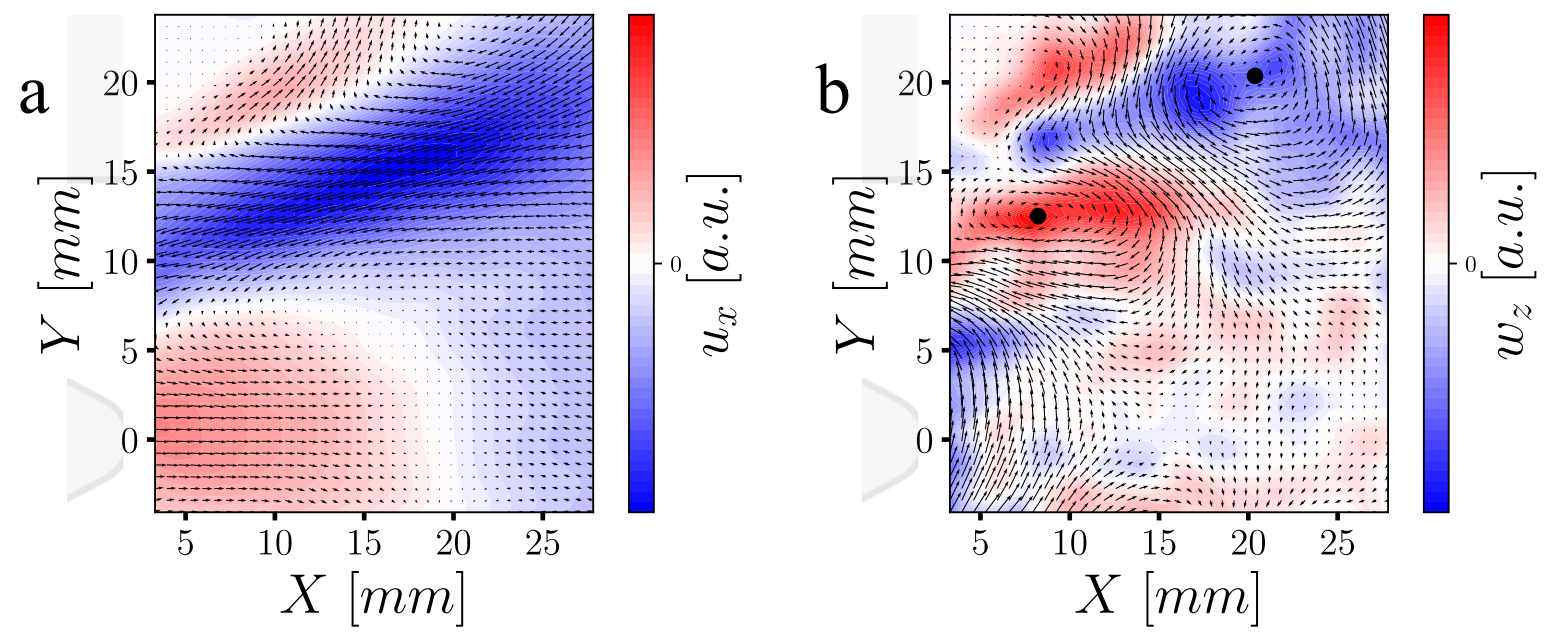

Figure 4: (a) Real portion of the $455 \mathrm{~Hz}$ DMD mode. Velocity vectors overlaid on filled contours of axial velocity. (b) Real portion of the $1704 \mathrm{~Hz}$ DMD mode with the center of the two counter-rotating vortices annotated by $\bullet$. Velocity vectors overlaid on filled contours of in-plane vorticity. Units in the colorbars are the unscaled DMD spatial mode values. 

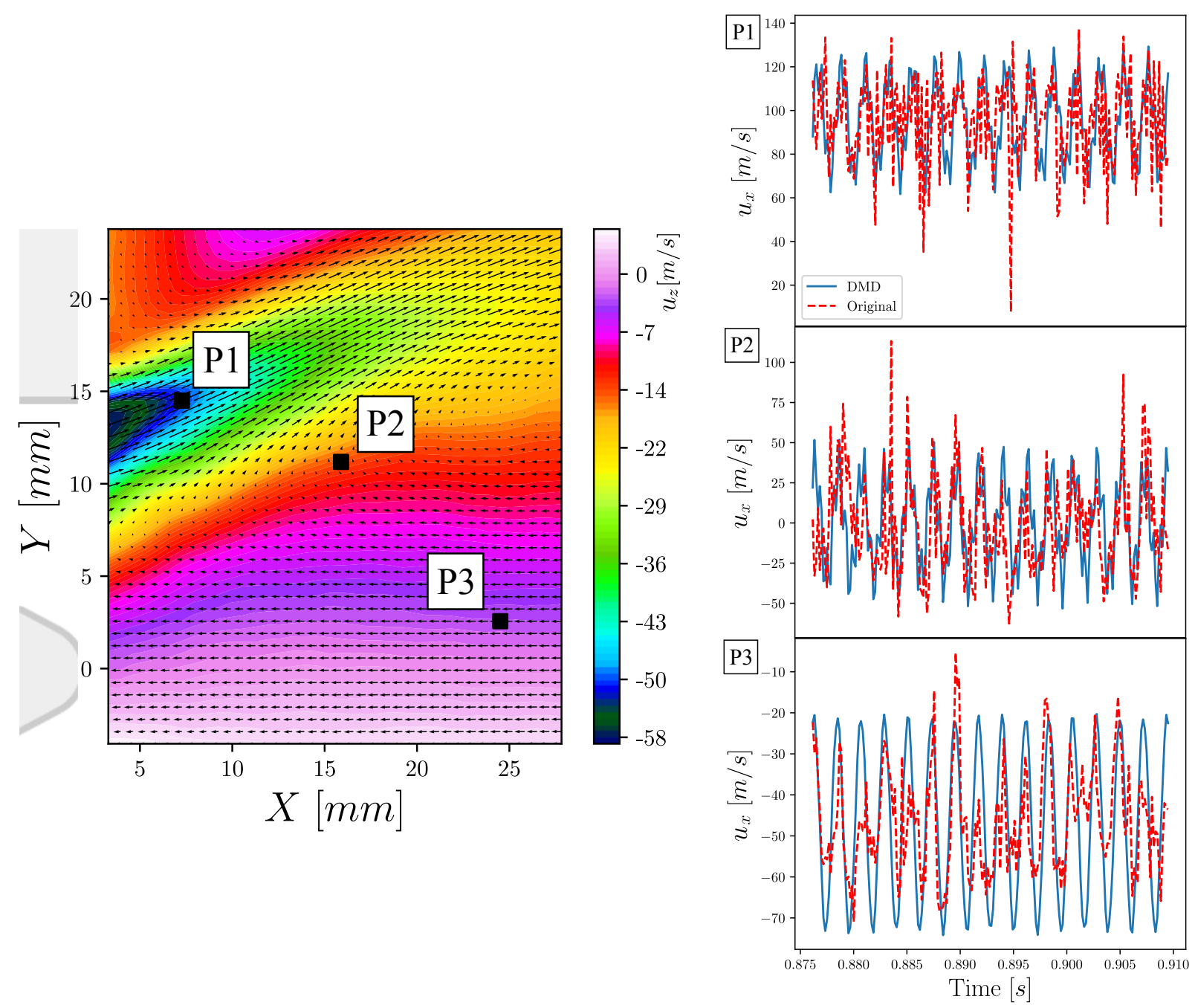

Figure 5: Average velocity field with contours of $u_{z}$. The DMD reconstruction and the original velocity field is compared at three probe points. 

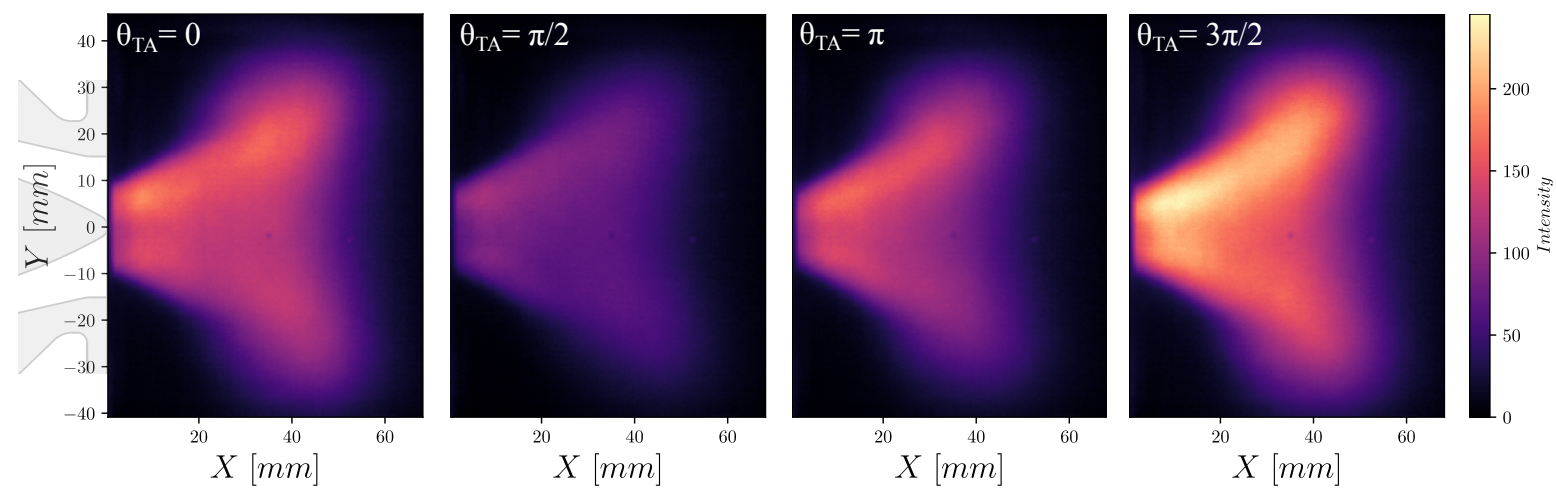

Figure 6: Phase average of $O H^{*}$ chemiluminescence images through the thermoacoustic cycle. 


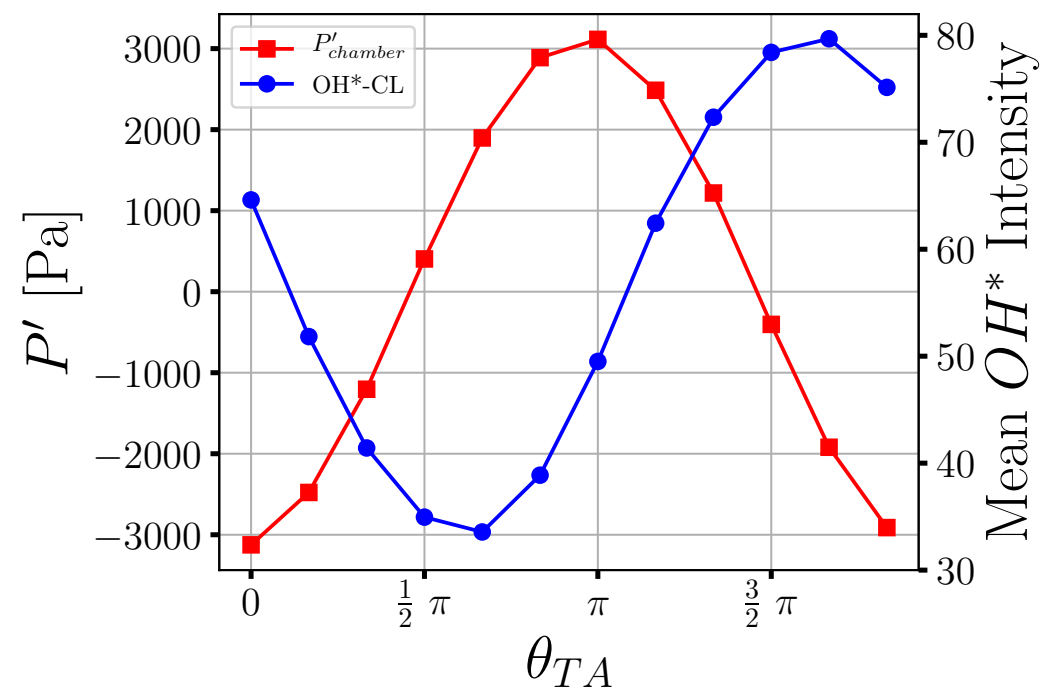

Figure 7: Phase average of mean $O H^{*}$ intensity and chamber dynamic pressure. 

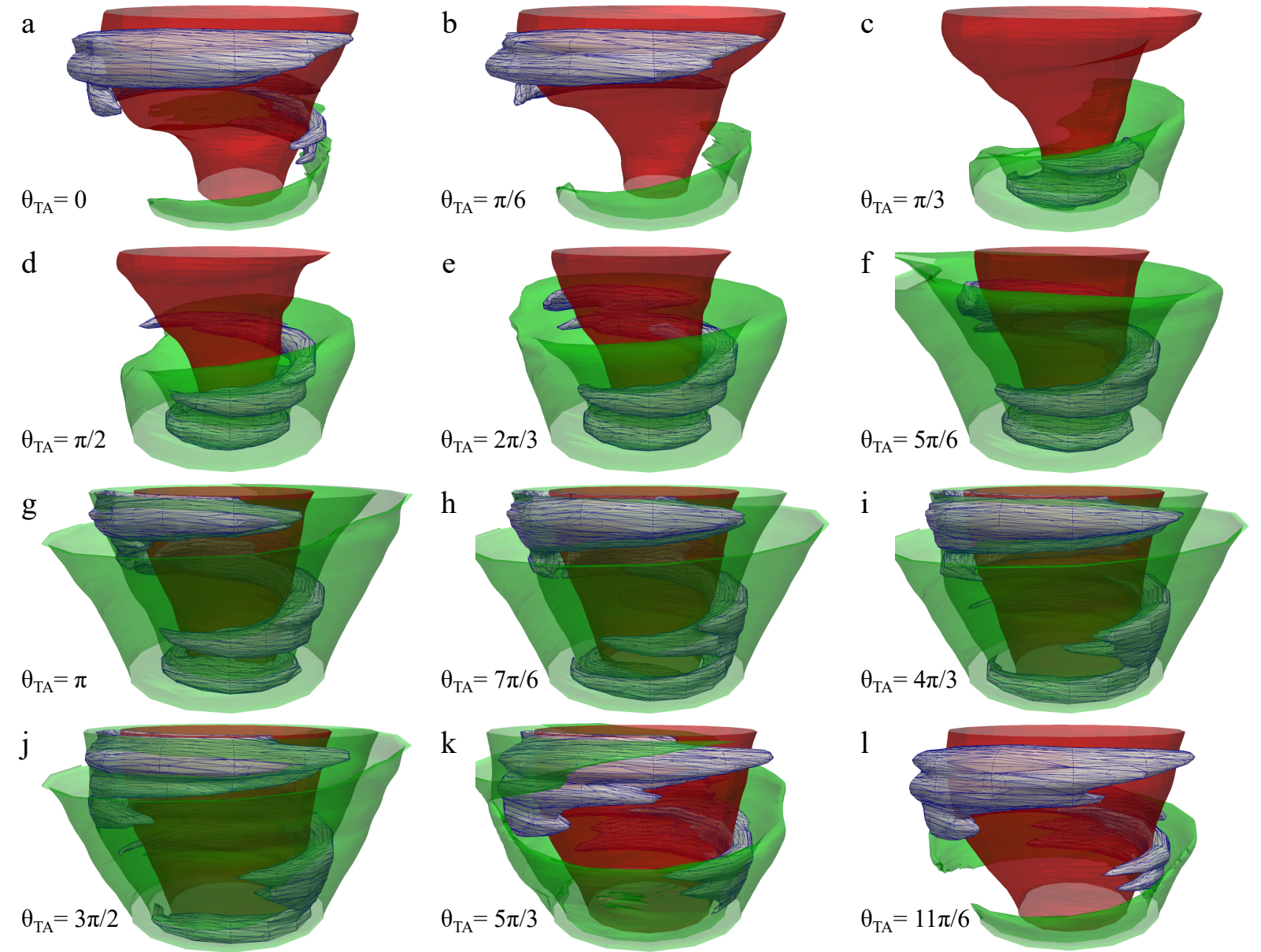

Figure 8: Flow structure evolution through the thermoacoustic cycle. The red and green iso-surfaces are $u_{x}=0 \mathrm{~m} / \mathrm{s}$ and $u_{x}=80 \mathrm{~m} / \mathrm{s}$, respectively. The gray and outlined iso-surface corresponds to the PVC defined as $\lambda_{c i}=8000 / \mathrm{s}$. 


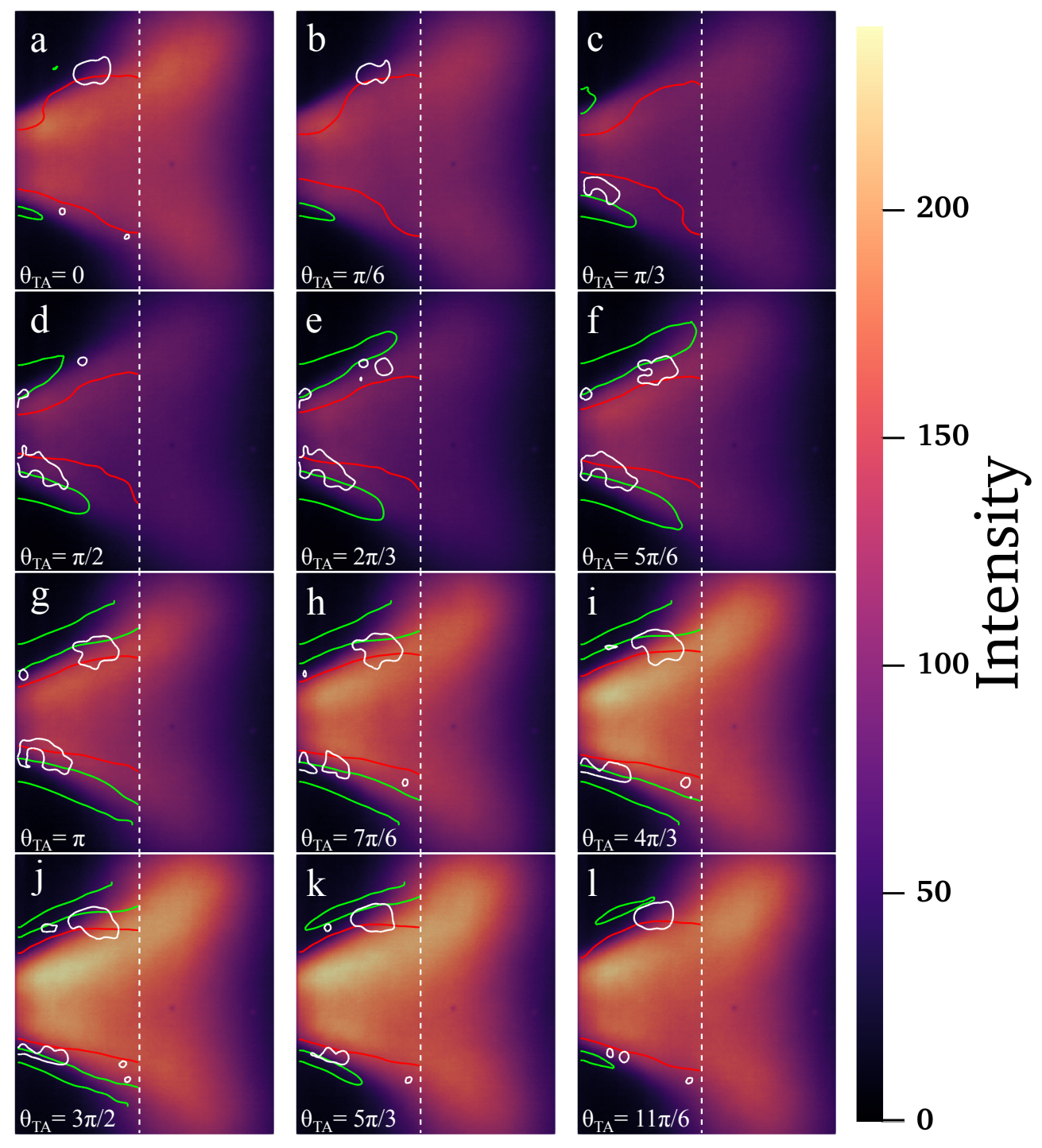

Figure 9: Overlaid 2D slices of the flow structures and the $\mathrm{OH}^{*}$-CL intensity distribution over the thermoacoustic cycle. The red and green contours are $u_{x}=0 \mathrm{~m} / \mathrm{s}$ and $u_{x}=80 \mathrm{~m} / \mathrm{s}$, respectively. The white contours corresponds to the PVC defined as $\lambda_{c i}=8000 / s$. Dotted white lines denote the maximum axial extent of the velocity measurements corresponding to figure 1 . 


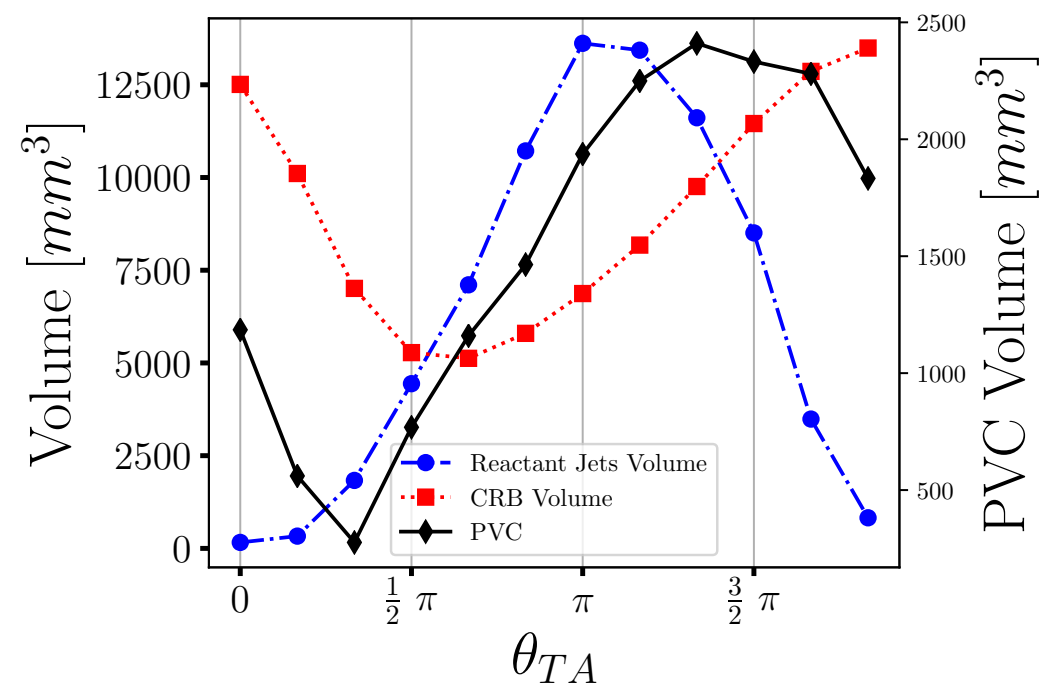

Figure 10: Volume of reactant jets, CRB, and PVC with $\theta_{T A}$ phase angle. 


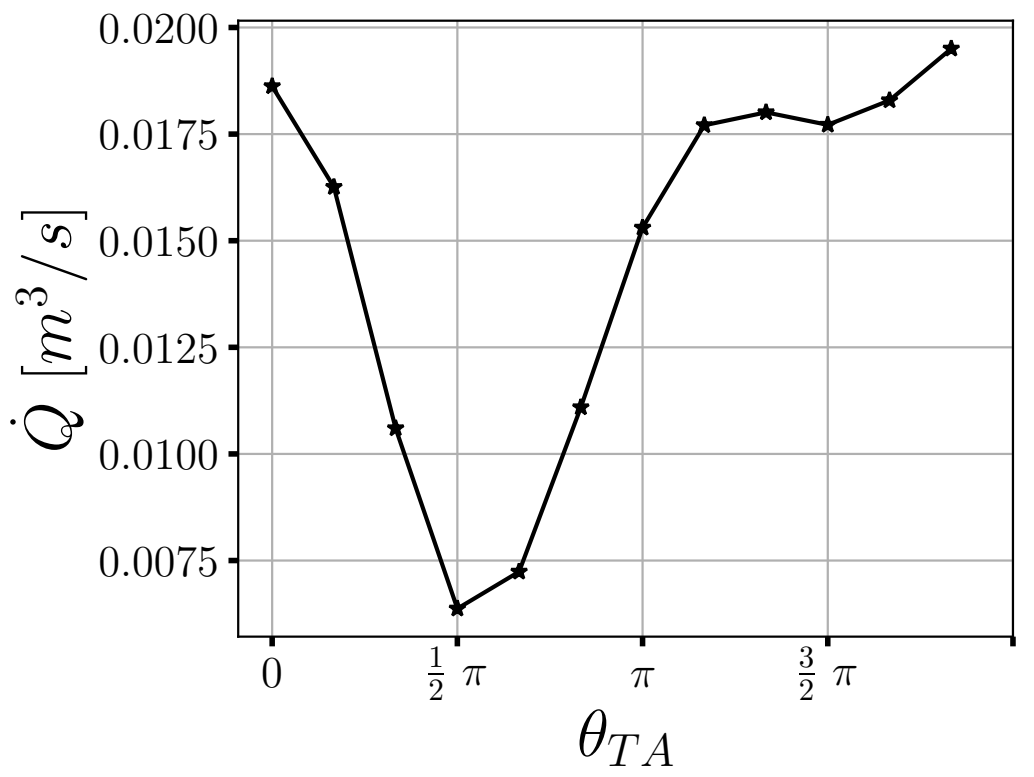

Figure 11: Volumetric flow rate out of the CRB throughout the thermoacoustic cycle. 


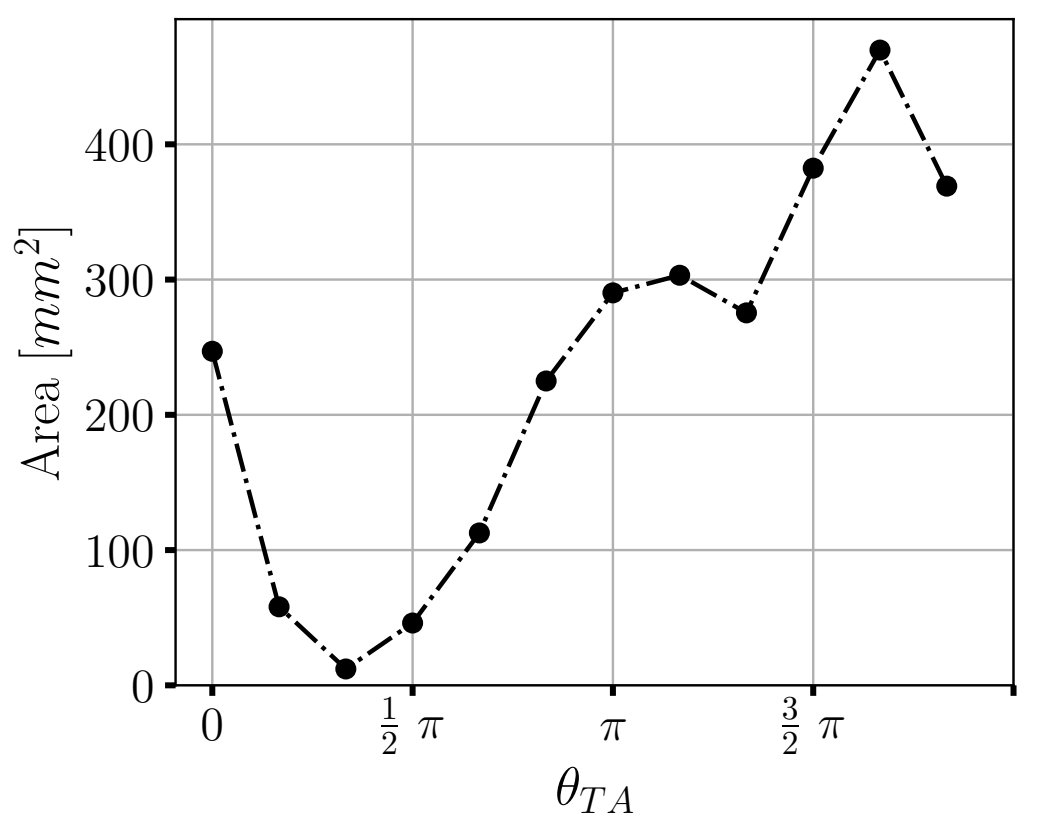

Figure 12: CRB surface area with $\lambda_{c i} \geq 8000 / s$. 


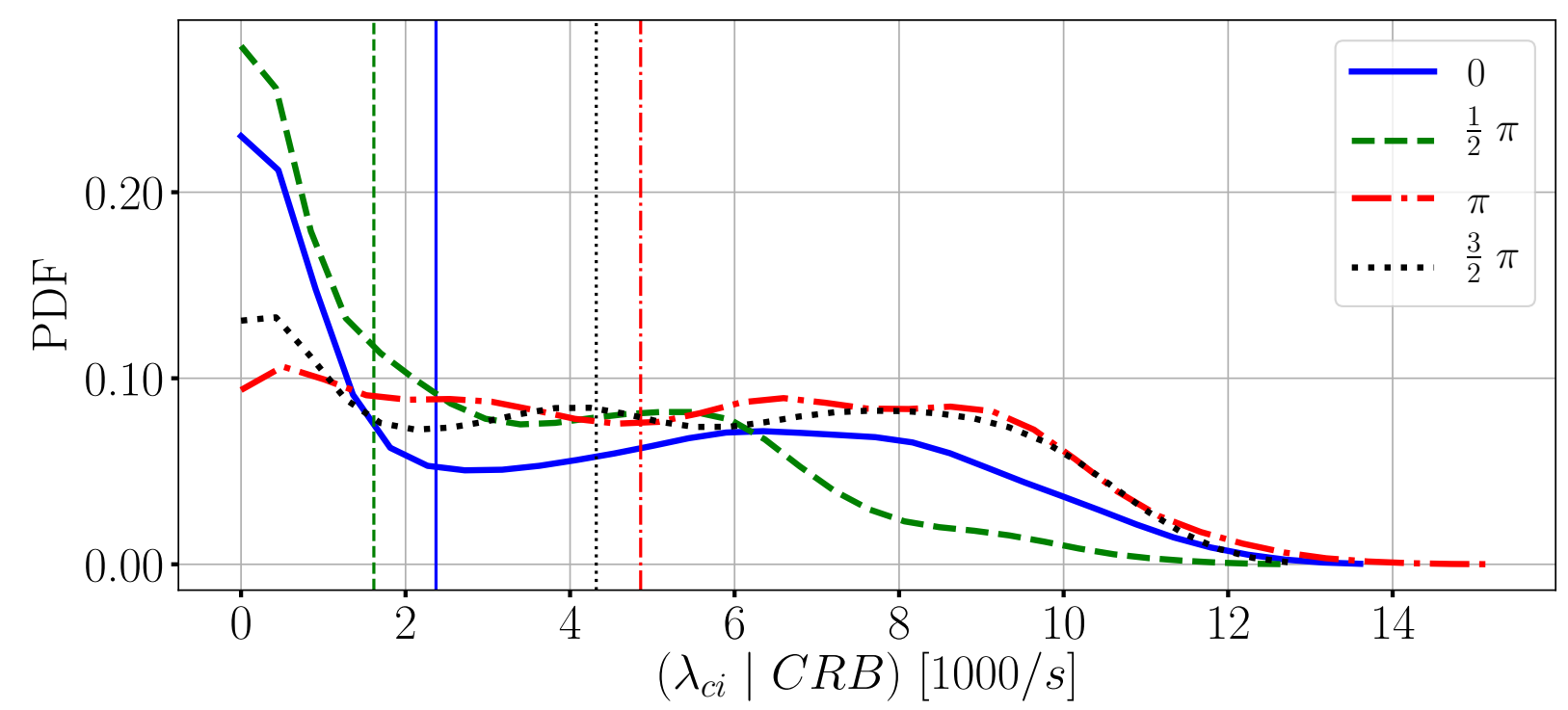

Figure 13: PDF of swirling strength on the CRB surface. Median values are shown by vertical lines. 

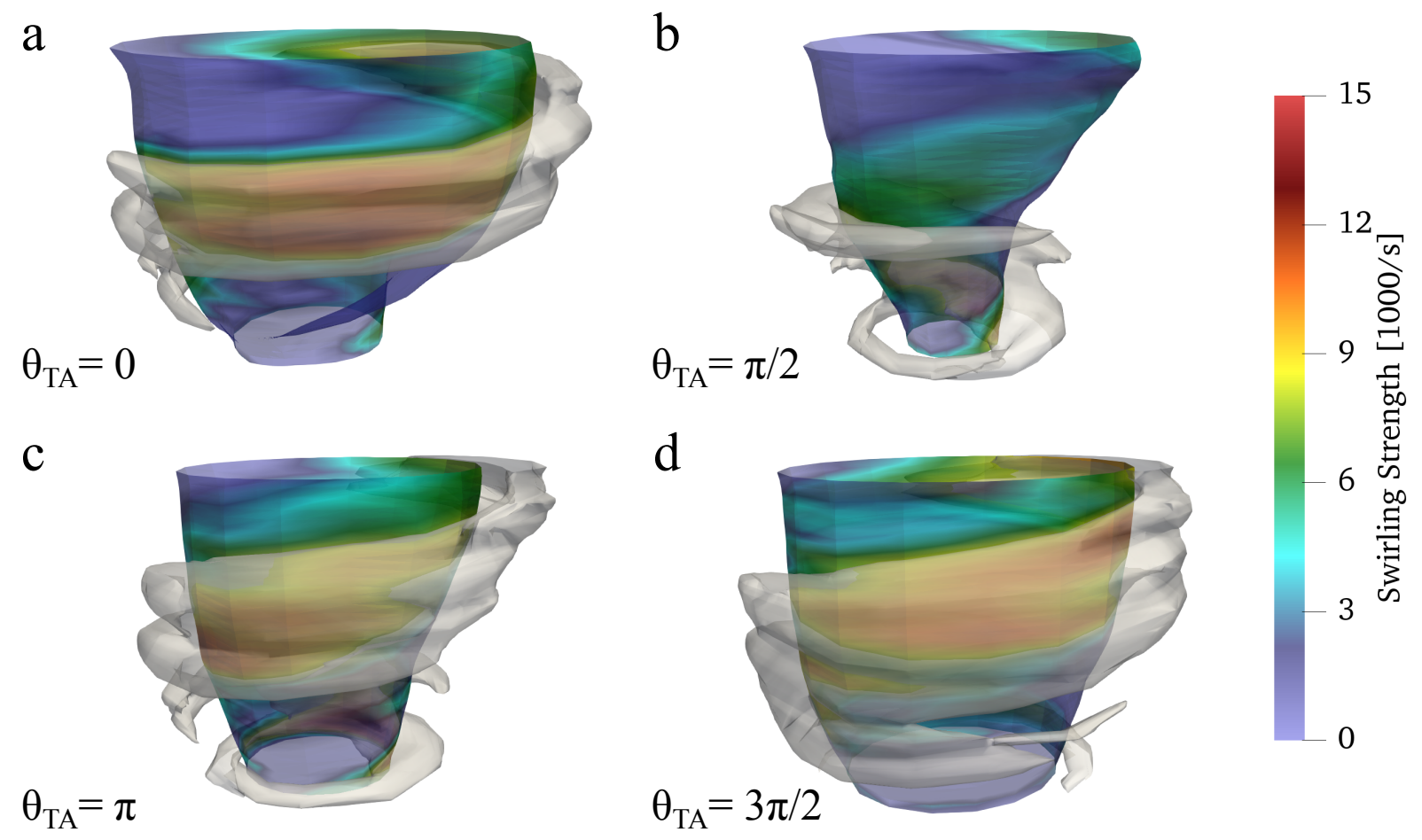

Figure 14: Surface of the PVC and CRB colored by $\lambda_{c i}$ at four phase angles of the TA cycle. 


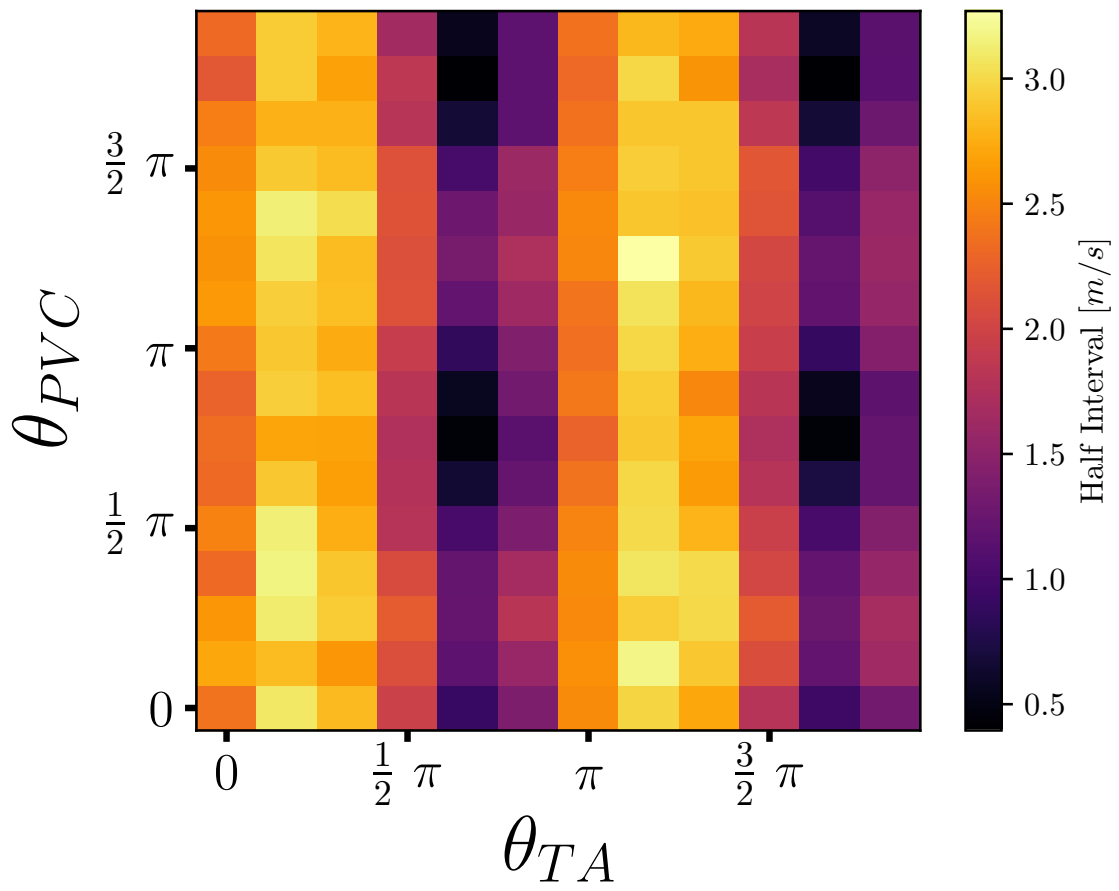

Figure 15: Confidence half intervals at each phase bin for the $u_{x}$ velocity component at P2 corresponding to the $12 \times 16$ phase bin case. 


\section{List of Tables}

1 Confidence Half Intervals of $u_{x} \ldots \ldots \ldots$. . . . . . . . . . . 37 
Table 1: Confidence Half Intervals of $u_{x}$

\begin{tabular}{c|ccc} 
Probe & Min & Max & Mean \\
\hline$P 1_{D M D-12 \times 16 \text { bins }}$ & 0.24 & 2.30 & 1.39 \\
$P 2_{D M D-12 \times 16 \text { bins }}$ & 0.40 & 3.27 & 2.08 \\
$P 3_{D M D-12 \times 16 \text { bins }}$ & 0.52 & 2.27 & 1.44 \\
$P 1_{D M D-4 \times 4 \text { bins }}$ & 0.40 & 1.76 & 1.15 \\
$P 2_{D M D-4 \times 4 \text { bins }}$ & 1.70 & 2.13 & 1.92 \\
$P 3_{D M D-4 \times 4 \text { bins }}$ & 0.57 & 1.74 & 1.15 \\
$P 1_{\text {Raw-8 bins }}$ & 1.59 & 2.53 & 2.07 \\
$P 2_{\text {Raw }-8 \text { bins }}$ & 2.83 & 3.84 & 3.42 \\
$P 3_{\text {Raw-8 bins }}$ & 1.22 & 1.56 & 1.44
\end{tabular}

